\title{
LUCIANA MASTANDREA
}

\section{Avaliação da marcha em idosas ativas e sedentárias}

Dissertação apresentada à Faculdade de Medicina da Universidade de São Paulo para obtenção do título de Mestre em Ciências Área de concentração: Ortopedia e Traumatologia

Orientadora: Prof ${ }^{\mathrm{a}}$ Dr ${ }^{\mathrm{a}}$ Júlia Maria D’Andrea Greve

São Paulo

2008 


\section{LUCIANA MASTANDREA}

\section{Avaliação da marcha em idosas ativas e sedentárias}

Dissertação apresentada à Faculdade de Medicina da Universidade de São Paulo para obtenção do título de Mestre em Ciências

Área de concentração: Ortopedia e Traumatologia

Orientadora: Prof ${ }^{a}$ Dr ${ }^{\mathrm{a}}$ Júlia Maria D’Andrea Greve

São Paulo

2008 


\section{DEDICATÓRIA}

Aos meus pais, Maria Angélica e Vicente, e ao meu marido, Sandro, pelo exemplo de dignidade, solidariedade, amor e dedicação. 


\section{AGRADECIMENTO ESPECIAL}

Ao Prof $^{\circ}$ Dr. José Maria Santarem Sobrinho, meu pai de profissão, que me co-orientou, incentivou, desde meus primeiros passos na área de atividade física. Minha admiração, carinho e eterna gratidão pelas oportunidades.

À Prof ${ }^{a}$ Dra. Júlia Maria D’Andrea Greve, que confiou em mim e sempre teve muita paciência e dedicação. Exemplo de uma grande mulher e profissional, que com propriedade conduziu esta orientação. Obrigada por tudo o que me foi ensinado.

\section{AGRADECIMENTOS}

Ao Prof $^{\circ}$ Dr. Wilson Jacob Filho, com quem aprendi a ter paixão pela minha área de atuação, mostrando que é com sabedoria, respeito, humanidade e dedicação que se conduz um trabalho.

À Prof ${ }^{a}$ Dra. Érica Maria Machado Santarem, pelo auxílio, postura e amizade, sempre aberta a me ouvir.

A minhas amigas Lidiane e Alcídia Ana Mulinário Lacerda (Diana) que me auxiliaram e acrescentaram muito no desenvolvimento deste trabalho. 
Aos colegas do Laboratório de Estudos do Movimento do Instituto de Ortopedia e Traumatologia do Hospital das Clinicas USP, em especial à Marlete e Narcísio, pelo auxílio neste trabalho.

À amiga Paula Maria Loiola de Souza, pela amizade, incentivo, auxílio e disposição que tiveram em meu trabalho.

À amiga Kátia Moura Rosa, que sempre acreditou em mim em todos os momentos, inclusive os difíceis, me animou, me incentivou e acompanhou a realização deste trabalho.

A todos os meus colegas e amigos do Instituto Biodelta, em especial ao Serginho, Edna, Flávia, Karen, Adriana, Fernanda, Sandra, Hélena e Carlos, pelo auxílio e suporte no instituto, no decorrer deste trabalho.

À Janaina Machado de Andrade, que como uma grande amiga me ouviu e me acolheu nos momentos difíceis. Só uma amiga faz tudo isso.

Ao meu marido e amigo Sandro Sargentim, pelo amor, amizade, respeito, torcida e incentivo. Obrigada pela paciência e pelo apoio constante em todos os momentos difíceis que passei.

Aos meus pais, Maria Angélica e Vicente, por todos os sacrifícios realizados para que eu pudesse concluir meus 
estudos. Muito obrigado pelo amor e carinho que sempre me deram e pelo incentivo em minha profissão.

A minha mãe, modelo de mulher e grande amiga, Maria Angélica Belloti Mastandrea, pelas palavras encorajadoras e apoio em toda a minha vida.

A minha sogra Dayse Crecchi Sargentim, pelo incentivo em minha carreira, que me abraçou como uma filha e tem me ensinado muito.

Ao meu sogro Hermínio Sargentim, pelo auxílio, respeito e dedicação que teve para comigo e para com meu trabalho.

Ao meu irmão Murillo, esposa Karla, obrigada pela torcida e carinho.

Aos meus queridos avós Vicentina Lofrano Belloti e Victório Belloti, minhas inspirações, que me ensinaram o que é viver e envelhecer com saúde. Responsáveis pelo meu amor e admiração pelos idosos.

A toda a minha família (tios, primos e amigos), muitas vezes distante, mas sempre torcendo pela minha realização.

Às idosas que participaram desta pesquisa, muito obrigada pela colaboração. Meu eterno carinho e respeito. 
"Aprender é ambíguo, significando ao mesmo tempo compreender usando o conhecimento, e adquirir conhecimento".

Aristóteles

(Os pensadores, 1999) 


\section{NORMALIZAÇÃO ADOTADA}

Esta dissertação está de acordo com as seguintes normas, em vigor no momento desta publicação:

Referências: adaptado de International Committee of Medical Journals Editors (Vancouver).

Universidade de São Paulo. Faculdade de Medicina. Serviço de Biblioteca e Documentação. Guia de apresentação de dissertações, teses e monografias. Elaborado por Anneliese Carneiro da Cunha, Maria Julia de A. L. Freddi, Maria F. Crestana, Marinalva de Souza Aragão, Suely Campos Cardoso, Valéria Vilhena. $2^{a}$ ed. São Paulo: Serviço de Biblioteca e Documentação, 2005.

Abreviaturas dos títulos dos periódicos de acordo com List of Journals Indexed in Index Medicus. 


\section{SUMÁRIO}

Lista de figuras

Lista de tabelas

Resumo

Summary

1 INTRODUCÃO 1

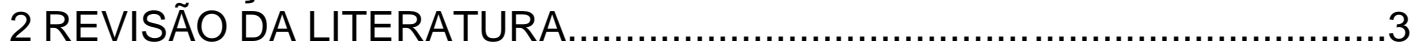

2.1 Envelhecimento e incapacidade funcional.........................................

2.2 Alteração da marcha no envelhecimento............................................

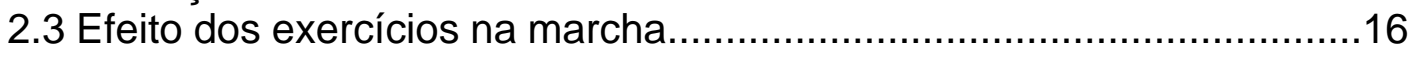

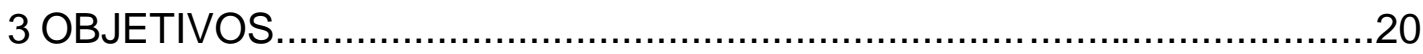

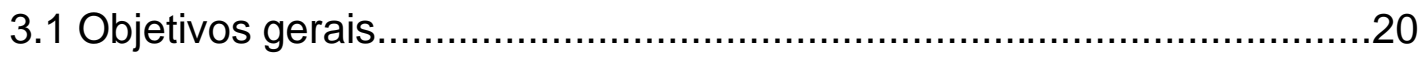

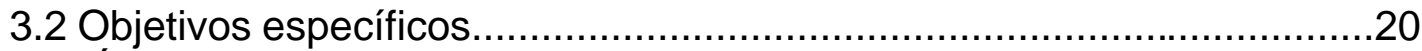

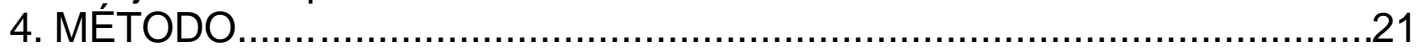

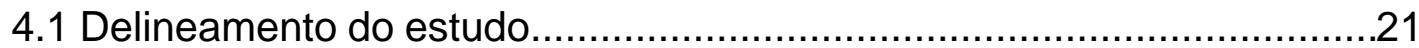

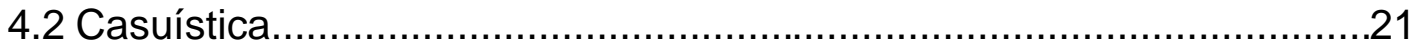

4.2.1 Critérios de inclusão...............................................................22

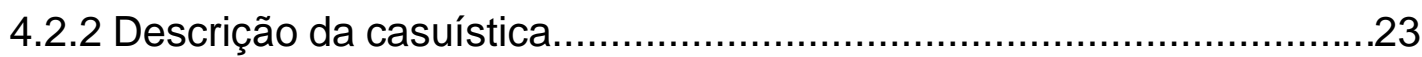

4.3 Metodologia de treinamento.......................................................24

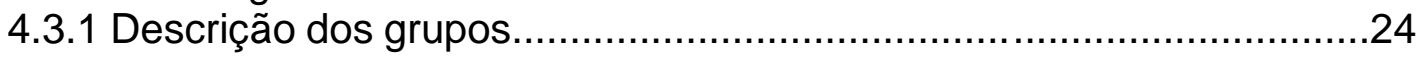

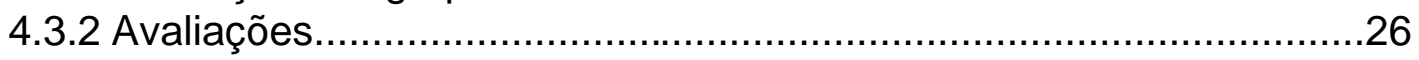

4.3.2.1 Avaliação da massa corporal e altura............................................26

4.3.2.2 Avaliação do tempo e da velocidade da marcha.............................26

4.3.2.3 Avaliação do comprimento do passo e da passada e largura do

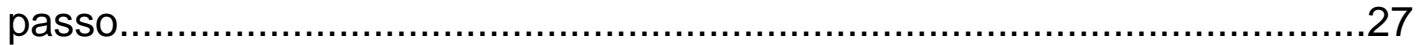

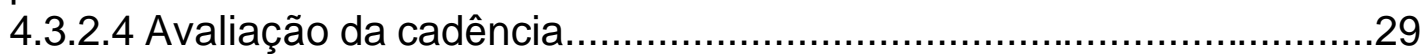

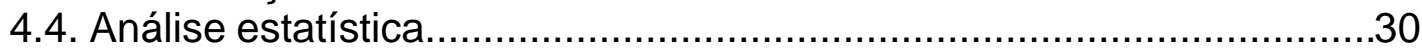

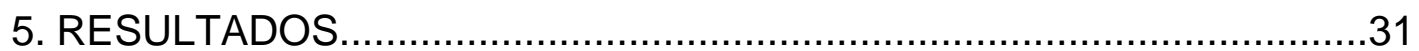

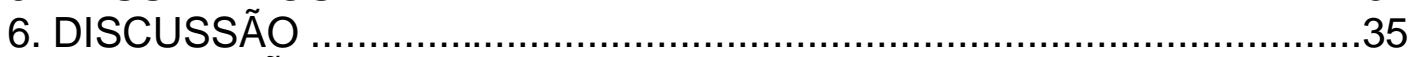

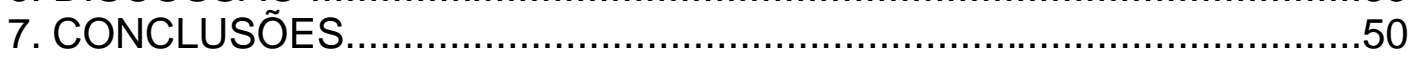

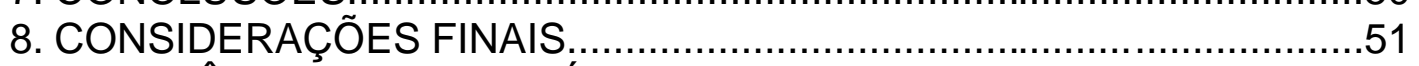

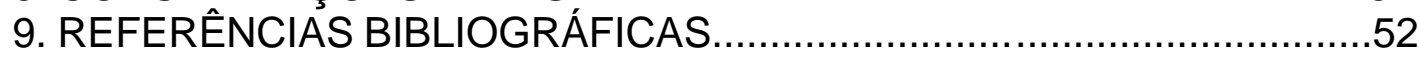

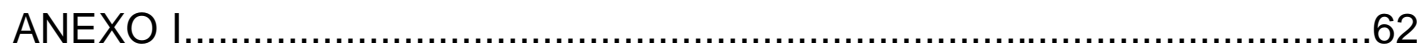

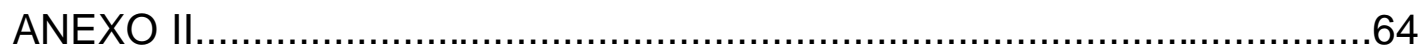

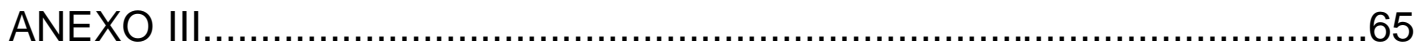

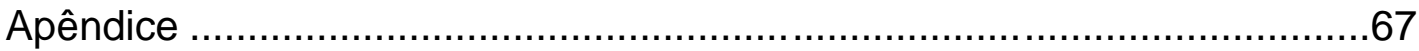




\section{LISTA DE FIGURAS}

Figura 1. Medida do tempo (segundos) e da velocidade de caminhada $(\mathrm{m} / \mathrm{s})$

em 4 e 10 metros .27

Figura 2. Pegadas com tinta azul em formulários contínuos de papéis brancos 28

Figura 3. Modelo utilizado para medição da largura e comprimento do passo e da passada (medido do centro do calcanhar) .29

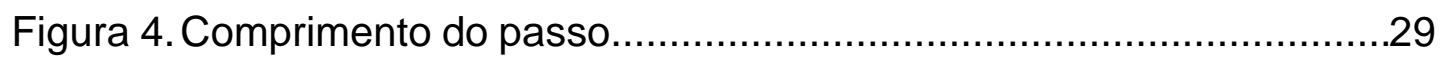

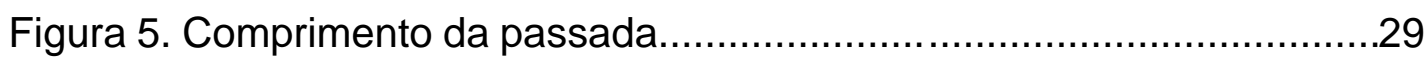

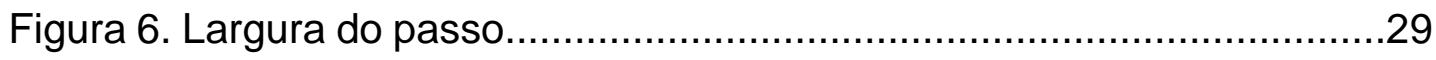




\section{LISTA DE TABELAS}

Tabela 1. Dados antropométricos (média e desvio padrão) dos indivíduos avaliados por grupo 23

Tabela 2. Média e desvio padrão do tempo de marcha (segundos) medido em 4 metros, 10 metros e 10 metros, com tinta nos pés, nos grupos Gerais, Resistidos e Sedentários 31

Tabela 3. Média e desvio padrão da velocidade da marcha (metros/segundo) em 4 metros, 10 metros e 10 metros, com tinta nos pés, nos Grupos Gerais, Resistidos e Sedentários 31

Tabela 4. Média e desvio padrão do comprimento dos passos e passadas (centímetros) medido em seis e em dez passos e passadas consecutivos, nos grupos Gerais, Resistidos e Sedentários. .33 Tabela 5. Média e desvio padrão da largura do passo (centímetros) em seis e dez passos consecutivos, nos grupos Gerais, Resistidos e Sedentários 34

Tabela 6. Média e desvio padrão da cadência (número de passos/minuto) em 10 metros, com tinta nos pés, nos grupos Gerais, Resistidos e Sedentários 


\section{LISTA DE QUADROS}

Quadro 1. Descrição dos resultados de autores diferentes e dos três grupos presentes no estudo com 60 a 69 anos... .45 
MASTANDREA, L. Avaliação da marcha em idosas ativas e sedentárias. Dissertação de Mestrado em Ciências. São Paulo: Faculdade de Medicina, Universidade de São Paulo, 2008.

\section{RESUMO}

O aumento da expectativa de vida é um dos objetivos da saúde pública, porém, não menos importante, é a preservação da capacidade e independência funcional. A dificuldade de locomoção é um dos fatores mais limitantes das atividades de vida diária dos idosos. O envelhecimento e a inatividade contribuem para a diminuição da força muscular e da flexibilidade, aumento da incidência de quedas e perda da capacidade para as atividades da vida diária. $\mathrm{O}$ objetivo deste estudo foi avaliar a marcha de indivíduos idosos: sedentários e praticantes de exercícios, gerais ou resistidos. Foram feitas as medidas espaciais e temporais da marcha com utilização de pegadas com tinta marcadas em uma passadeira de papel e de um cronômetro. Os parâmetros avaliados foram medidos em marcha de dez metros. Resultados: O grupo de sedentários apresentou menor velocidade e maior tempo da marcha; menor comprimento dos passos e das passadas, e menor cadência. Entre os dois grupos praticantes de exercícios, não houve diferenças. A largura do passo não foi diferente entre os três grupos, pois depende mais dos fatores antropométricos para 0 posicionamento dos pés. Os exercícios físicos podem ter influenciado nos parâmetros da marcha do que a altura dos indivíduos. Este estudo mostra que o exercício físico regular melhora os parâmetros da marcha.

Palavras-chave: idoso, marcha, exercício, mulheres, caminhada, envelhecimento. 
MASTANDREA, L. Assessment of gait in physically active and sedentary elderly women.

Dissertation in Master of Science. Sao Paulo: Faculty of Medicine, University of Sao Paulo, 2008.

\section{SUMMARY}

The rise of life expectancy is one of the objectives in public health but not less important is the preservation of the capacity and functional independence. The locomotion difficulty is one of the most limiting factors of the elderly daily activities (ADL). Ageing and inactivity contribute to the decrease of the muscular strength, flexibility, functional capacity an falls. The purpose of this study was to evaluate the gait of elderly subjects: sedentary, practitioners of general exercises and practitioners of resistance exercises. Spatial and temporal measures have been taken from the gait with the use of footprints with marked paints on a paper walkway and a stopwatch. The parameters evaluated were measured in gait of 10 meters. Results: the sedentary group presented lower gait speed, shorter length of steps and strides and decrease of the cadence.

There were no differences between the exercises group. There was no difference in the step width among the three groups. Physical exercises have more influence on the gait parameters than the subjects' height. This study demonstrates that regular physical exercise improve the gait parameters.

Key words: aged, gait, exercise, women, walking, aging. 


\section{INTRODUÇÃO}

A Organização Mundial da Saúde (OMS), na era do envelhecimento global, manifesta reiteradamente sua preocupação com o aumento da expectativa de vida, considerando, principalmente, o assustador aspecto da incapacidade e da dependência, as maiores adversidades da saúde associadas ao envelhecimento (Freitas et al., 2002).

Segundo Miller (1993), apud Frontera (2001), "o envelhecimento é um processo que converte adultos saudáveis em adultos frágeis, com reservas diminuídas em muitos sistemas fisiológicos e uma vulnerabilidade exponencialmente crescente para muitas doenças e para a morte". 0 aumento da expectativa de vida trouxe grandes perspectivas para as pessoas desfrutarem plenamente a sua "melhor idade".

O Brasil era tido como um país de jovens até o século XX. Contudo, a partir da década de 60 , a população brasileira vem envelhecendo. $O$ índice de envelhecimento passou de 0,11 (início da década 80), para 0,25, em 2004, ( 25 idosos para cada 100 jovens), . No Brasil entre 1980 e 2020, segundo Chaimowicz (1997) e Garrido \& Menezes (2002), o número de idosos com 60 anos ou mais de idade aumentará $280 \%$. O envelhecimento populacional iniciou-se na década de 1960 pela diminuição da natalidade associada à queda da mortalidade nas regiões mais desenvolvidas (Chaimowicz, 1997 e Garrido \& Menezes, 2002). As projeções populacionais das Nações Unidas para 2005 apontam que o Brasil pertence ao grupo dos dez países com maior número de pessoas de 60 anos ou mais, em termos absolutos, em sua população (IBGE, 2006). O processo de envelhecimento 
populacional brasileiro está ocorrendo mais rápido do que nos países desenvolvidos e há necessidade de se adequar o sistema de saúde para a atender esta população.

A fragilidade física resulta da interação entre envelhecimento, acúmulo de doenças crônicas e estilo de vida, em particular o sedentarismo (Frontera et al., 2000). Com o envelhecimento, ocorre diminuição progressiva da massa muscular (sarcopenia), da força e flexibilidade, o que contribui para a ocorrência de quedas (Fiatarone et al., 1990; Thompson et al., 1994; Guralnick et al., 1995; Frontera et al., 2000; Roth et al., 2001) e diminui a capacidade de realização das atividades da vida diária (AVDs). A independência funcional está relacionada à manutenção da mobilidade e da qualidade de vida. As perdas funcionais do equilíbrio e da marcha são os principais fatores de risco para limitação da mobilidade e quedas entre os idosos (Daley \& Spinks, 2000) Há evidências de que o exercício físico pode reverter incapacidades físicas e melhorar o desempenho para as tarefas diárias, como o tempo de caminhada.

Para o idoso, a dificuldade de locomoção é um dos fatores que mais o limita nas atividades diárias. Sendo assim, a avaliação da marcha é um importante elemento para avaliação geral de um idoso.

O objetivo deste estudo é avaliar a marcha de indivíduos idosos sedentários e praticantes de exercícios gerais (exercícios com predominância aeróbia, calistênicos, de flexibilidade e resistidos de baixa intensidade) e exercícios resistidos (musculação com equipamentos) usando os parâmetros espaciais e temporais da marcha. 


\section{REVISÃO DA LITERATURA}

\subsection{Envelhecimento e incapacidade funcional}

O processo de envelhecimento pode ocorrer de duas maneiras:

- envelhecimento fisiológico (senescência) - reduções fisiológicas não agravadas por doenças, estilo de vida ou ambiente;

- envelhecimento patológico (senilidade) - causado pelos efeitos combinados do envelhecimento fisiológico com as doenças e fatores adversos do estilo de vida e do ambiente (Papaléo Neto \& Carvalho Filho, 1996).

O "Estudo de Incapacidades de Framinghan" (Framinghan Disability Study, 2001) mostrou que o risco de incapacidade física aumenta com a idade. Vinte e cinco por cento das pessoas com idade superior a 65 anos não eram capazes de levantar 5 quilos ou caminhar 500 metros (Jette \& Branch, 1981, apud Frontera, 2001). Para muitos indivíduos, o funcionamento independente pressupõe a habilidade para caminhar (Bohannon et al., 1997).

A limitação funcional é menor nas mulheres que nos homens, entre 60 e 74 anos e se iguala aos 75 anos. Os idosos têm mais incapacidades com relação a limitações físicas do que os jovens (Daley \& Spinks, 2000). As alterações, inerentes ao processo de envelhecimento, que afetam 0 equilíbrio e a marcha são: enrijecimento tecido conectivo (perda da flexibilidade e amplitude de movimento articular); hipotensão postural, 
redução da capacidade aeróbia, acúmulo de tecido adiposo, alterações vestibulares e visuais, hipoacusia e as doenças crônico-degenerativas: cardiovasculares, neurológicas, alterações sensoriais e músculoesqueléticas (Daley \& Spinks, 2000; Marsico et al., 2002).

Os músculos das pessoas idosas tem grande atrofia, pela perda de fibras do tipo II, e comprometimento da força (Fiatarone et al., 1990; Frontera et al., 1998; Freitas et al., 2002). A sarcopenia do envelhecimento, agravada pelo sedentarismo, causa perda de força e mobilidade, e alterações na marcha e equilíbrio (Fiatarone et al., 1990; Guralnick et al., 1995; Frontera et al., 2000; Judge et al., 2003; Muhlberg \& Sieber, 2004). A força e a massa muscular declinam de 30 a 50\% entre 30 e 80 anos de idade. Uma pessoa sedentária perde de 5 a 10\% da massa muscular entre os 20 e 50 anos e 30 a $40 \%$ entre os 50 e 80 anos. Este declínio é maior nas mulheres (Daley \& Spinks, 2000; Judge et al., 2003).

O tamanho do músculo, a força e a habilidade para realizar as atividades da vida diária alcançam o pico aos 30 anos e declinam com a idade. Este declínio ocorre independentemente do condicionamento físico ou da quantidade de treinamento (Landers et al., 2001).

A deterioração da função sensório-motora do músculo contribui para o medo de andar, de ficar em pé e nas quedas, com expressiva perda de independência (Hurley et al., 1998).

Há uma relação entre o nível de independência funcional e a manutenção da capacidade de marcha. Autores como Guralnick et al. (1995), Ostir et al. (2002) e Steffen et al. (2002) sugerem que a marcha é um 
bom indicador da perda de autonomia com o envelhecimento. Guralnick et al. (1994) testaram a função de membros inferiores em cinco mil pessoas, com idade de 71 anos ou mais. Os testes se baseavam no equilíbrio, velocidade de marcha em 2,4 metros e força para se levantar de uma cadeira cinco vezes. Os testes de desempenho foram associados com o auto-relato de incapacidade e se constituíram em indicadores independentes de mortalidade e admissão em casas de repouso.

O processo de envelhecimento pode associar-se a modificações na forma de andar e a um maior tempo para percorrer certa distância (Hausdorff et al., 2001; Farinatti e Lopes, 2004). A intervenção com exercícios de fortalecimento muscular melhora a força nos membros inferiores e reduz a instabilidade da marcha (Prince et al., 1997; Hausdorff et al., 2001).

\subsection{Alterações da marcha no envelhecimento}

A marcha está relacionada com a maturidade neuromuscular e do desenvolvimento de habilidades motoras. (Prince et al., 1997; Sízinio e col., 1998; Rubino, 2002).

O equilíbrio dinâmico durante a marcha é controlado pela integração das aferências do sistema visual, vestibular e proprioceptivo sobre a o sistema musculo-esquelético. Uma pessoa saudável caminha com uma velocidade auto-selecionada, calibrada pelo custo energético. $\bigcirc$ envelhecimento reduz a velocidade e eficiência da marcha (maior consumo de oxigênio e fadiga) (Prince et al., 1997). 
O ciclo de marcha inicia-se quando o calcanhar de um membro a toca o solo e termina quando o calcanhar do mesmo membro toca novamente o solo (Sullivan, 1993; Perry, 2005). Segundo Sullivan (1993), Marsico et al. (2002) e Perry (2005), o ciclo da marcha é dividido em duas fases, apoio e balanço e dois períodos de duplo apoio. A fase de apoio constitui $60 \%$ do ciclo da marcha (toque do calcanhar, apoio inicial, médio apoio, apoio terminal e pré-balanço). Nessa fase, os músculos eretores espinhais mantêm a postura vertical, os glúteos máximos previnem a flexão de quadril e o tronco não se inclina em direção à coxa e o quadríceps mantém a extensão de joelho. A fase de balanço constitui $40 \%$ do ciclo da marcha (balanço inicial, balanço médio e balanço final). O período de duplo apoio é a fase em que os dois pés estão em contato com o solo (Marsico et al.,2002 e Perry 2005).

O comprimento do passo é a distância longitudinal entre o apoio do calcanhar de um membro no solo e o apoio do calcanhar contra-lateral no solo. O comprimento da passada é a distância entre o apoio do calcanhar de um membro no solo e a volta do apoio desse mesmo calcanhar no solo. A largura do passo é o distanciamento entre os pés. Estas são as variáveis espaciais. (Sullivan, 1993; Perry, 2005). A variável temporal da marcha é a velocidade, calculada pela divisão da distância percorrida pelo tempo dispendido. A cadência é o número de passos dados por minuto (Sullivan, 1993; Perry, 2005). A velocidade de marcha é uma medida válida e prática da mobilidade e reflete a atividade funcional da vida diária. É um indicador do declínio funcional e da fragilidade e está relacionada com risco de 
admissão nas casas de repouso e mortalidade (Judge et al., 1993; Oberg et al., 1993; Guralnick et al., 1994; Bohannon et al., 1997; Brach et al., 2001). Guralnick et al (2000) sugeriram um percurso padronizado de quatro metros para avaliar a velocidade de marcha e referem que a avaliação da marcha, sozinha, é tão boa quanto a bateria de testes de desempenho, validados pelos autores, na predição das incapacidades.

O envelhecimento leva ao aumento da cifose torácica, perda da lordose lombar e deslocamento anterior do centro de gravidade; anteriorização da cabeça; diminuição da capacidade de elevação do pé na fase de balanço, passos e passadas mais curtos, aumento do tempo de duplo apoio; perda do movimento de rolamento do pé (do toque do calcâneo ao despreendimento do hálux); diminuição da amplitude de movimento articular dos membros inferiores e redução do movimento dos braços e diminuição da velocidade da marcha normal (Leiper \& Craik, 1991; Elble et al., 1991; Ronsky et al., 1995; Henriksson \& Hirschfeld, 2005).

A manutenção da flexão de joelho, no final da fase de balanço, é causada pela perda da força de quadríceps, que também se associa aos passos mais curtos (Oberg et al., 1993; Ostrosky et al.,1994). A velocidade e o comprimento do passo diminui com a idade (Oberg et al. 1993, Bohannon et al. 1997, e Steffen et al. 2002 (Ostrosky et al., 1994; Alexander, 1996; Sekiya et al., 1997). Judge et al., 1993, 1996; Prince et al.,1997; Kerrigan et al., 1998 referem que o encurtamento dos passos está associada à diminuição da velocidade, porém com manutenção ou aumento da cadência, levando à perda e eficiência da marcha e às quedas. $\mathrm{O}$ 
decréscimo de $0,1 \mathrm{~m} / \mathrm{s}$ na velocidade da marcha está associado com 10\% de decréscimo na habilidade para as atividades da vida diária (Prince et al., 1997; Daley \& Spinks, 2000).

Elble et al. (1991) estudaram os parâmetros espaciais e temporais da marcha de 20 idosos (65 a 87 anos) e 20 jovens (20 a 30 anos), todos praticantes de exercícios regularmente, em percurso de 10 metros. A média de velocidade na marcha auto-selecionada foi $20 \%$ menor nos idosos. Em velocidade rápida foi $17 \%$ menor. Os autores referem que os idosos têm passos mais curtos, causadas pela perda de mobilidade articular e redução da potência muscular do quadril e do joelho.

Oberg et al. (1993) analisaram a marcha de 233 indivíduos saudáveis, com idades entre 10 e 79 anos, em um percurso de 10 metros. Referem que a idade e gênero influenciam na velocidade da marcha (auto-selecionada e rápida). O envelhecimento causa diminuição da velocidade da marcha e do comprimento dos passos. As mulheres andam em velocidade menor e com passos mais curtos. Bohannon et al. (1997) avaliaram 230 indivíduos saudáveis, de 20 a 79 anos, e concluíram que a velocidade da marcha está relacionada com a idade, altura e força muscular dos membros inferiores.

Ostrosky et al. (1994) analisaram os ângulos de quadril, joelho e tornozelo em marcha com velocidade auto-selecionada em jovens e idosos fisicamente ativos. O pico de extensão de joelho, o comprimento da passada e a velocidade foram significativamente menores nos idosos.

A redução da força dos flexores plantares correlaciona-se com o encurtamento dos passos e diminuição da velocidade de marcha em idosos. 
A redução da força dos flexores plantares causa maior flexão de quadril, para aumentar o comprimento do passo (Ronsky et al., 1995; Judge et al., 1996; Mcgibbon, 2003). Sekiya et al. (1997) mostram a correlação positiva entre aumento do comprimento dos passos e cadência e aumento de velocidade de marcha. A largura dos passos, também aumentou, de forma linear, com o aumento da velocidade. A largura do passo está relacionada à manutenção do equilíbrio.

Os idosos apresentam encurtamento do passo, na comparação com adultos jovens, em velocidade de marcha auto selecionada, pela diminuição da extensão de quadril, que é causada pela fraqueza muscular e encurtamento dos flexores do quadril (Kerrigan et al., 1998 e Riley et al. 2001).

Scarborough et al. (1999) avaliaram a estabilidade dinâmica para levantar-se da cadeira e caminhar oito metros em 34 idosos. Os idosos com mais força de quadríceps tiveram mais estabilidade e habilidade para controlar o movimento de levantar-se da cadeira e maior estabilidade dinâmica durante a marcha. O comprimento da passada, a velocidade de e o tempo de duplo apoio estão inversamente correlacionados com a força do quadríceps. Judge et al., (1996) demonstraram que a força do quadríceps apresenta significativa relação com o comprimento do passo, na avaliação de 26 idosos.

Stolze et al. (2000) testaram a validade e confiabilidade de um teste de marcha realizado em um percurso de 10 metros, utilizando tinta para marcar as pegadas dos pés em 22 mulheres jovens (21 a 37 anos) e 22 
mulheres idosas (64 a 92 anos). Avaliaram o padrão locomotor e parâmetros da passada em relação à idade. O comprimento da passada e a velocidade da marcha foram menores nos idosos. A largura do passo e o ângulo dos pés não variam com a idade. Este teste teve boa correlação com a avaliação cinemática feita por filmagens.

De Castro e col. (2000) avaliaram os parâmetros temporais e espaciais da marcha em velocidade auto-selecionada de 15 idosos, brasileiros, de ambos os gêneros, com idades entre 60 e 79 anos e, os compararam com os dados normativos de Oberg et al. (1993). A velocidade da marcha foi inferior no Brasil, fato atribuído à menor estatura e comprimento de passos.

Brach et al. (2001) estudaram os efeitos da variação de velocidade nos parâmetros espaciais e temporais da marcha de 95 idosos de ambos os sexos e verficarm que quanto menor a velocidade, maior a variabilidade de comprimento e largura dos passos.

Hausdorff et al. (2001) investigaram os fatores contributivos para a instabilidade de marcha nos idosos. Foram avaliados 67 indivíduos, idade superior a 70 anos, sedentários e de ambos os gêneros. Os autores concluíram que a fraqueza muscular e a limitação do movimento articular estão associadas com maior tempo de duplo apoio, encurtamento das passadas e perda de equilíbrio durante a marcha. A realização de um programa de exercícios resistidos, e equilíbrio e atividades aeróbias, três vezes por semana, por seis meses, trouxe melhora da força muscular e diminuição da instabilidade durante a marcha. 
Grabiner et al. (2001) avaliaram o efeito da idade, velocidade, condição de calçado e desempenho sob condição de atenção na marcha de indivíduos idosos e jovens. Os indivíduos andaram, com e sem sapatos, em três velocidades diferentes e caminharam com e sem uma xícara com água na mão. Nos dois testes, a largura dos passos foi maior nos idosos que nos jovens. O calçado, a condição de atenção e a velocidade da marcha não demonstram diferenças com relação à idade. Os idosos mostram um comprimento do passo mais regular quando comparado com os jovens e mais largo (41\% maior). Nas demais variáveis não se observou diferença. Os autores sugerem que as mudanças nas variáveis da marcha contribuem para as quedas nos idosos, pois muitas quedas ocorrem durante o início da marcha, numa fase de transição que requer maior sinergismo muscular.

Ostir et al. (2002) estudaram o equilíbrio, a caminhada de quatro metros e cinco repetições do movimento de levantar e sentar de uma cadeira. Foram avaliadas 102 mulheres (com incapacidade moderada a grave), separadas por grupos etários (65 a 74 anos; 75 a 84 anos e acima de 85 anos). O primeiro grupo (65-74 anos) teve melhor desempenho que os demais. Os resultados revelam que essas tarefas têm uma excelente confiabilidade e alta sensibilidade com relação à independência diária em pessoas idosas, pois avaliam a função física dos membros inferiores.

Rubino (2002) refere que a velocidade de marcha dos idosos pode ser aumentada pelo condicionamento físico. Segundo o autor, nos idosos, a linha de gravidade se desloca para trás, pela rotação pélvica e mudança da curvatura espinal, facilitando a queda para trás. 
As associações entre força de membros inferiores, velocidade e comprimento dos passos são vistas em vários estudos (Buchner et al., 1996; Judge et al., 1996; Bohannon et al., 1998; Lamoureux et al., 2002; Barret \& Smerdely et al., 2002; Menz et al., 2003) e indicam que o declínio da força muscular dos membros inferiores, causado pelo envelhecimento, pode ser um fator limitante primário para a manutenção da velocidade de marcha nos idosos.

O controle de equilíbrio durante a marcha é comprometido com o avanço da idade (Hausdorff et al., 2001). O aumento da variabilidade da largura dos passos está associado com a perda de habilidade motora e pode ser um indicador de quedas (Hausdorff et al., 2001; Grabiner et al., 2001; Owings \& Grabiner, 2003).

Owings \& Grabiner (2003) avaliaram a influência da idade (12 idosos e 18 jovens), da velocidade e do apoio das mãos durante uma caminhada na esteira. A caminhada na esteira pode ser uma representação aceitável da caminhada no solo. Há maior variabilidade da largura dos passos que do comprimento. Os passos dos idosos são mais largos e mais variáveis e esta variabilidade pode ser associada às quedas.

Helbostad \& Moe-nilssen (2003) investigaram a relação entre velocidade da marcha com largura do passo, variabilidade da largura do passo e aceleração do tronco. Foram incluídos 36 indivíduos praticantes de exercícios físicos uma vez por semana, de ambos os sexos, com idades entre 66 a 79 anos. No percurso de 10 metros, em quatro velocidades diferentes não houve relaçã intra-indivíduos. A aceleração do tronco é menor 
em indivíduos mais altos e maior nos mais pesados. A largura do passo é menor nas mulheres do que nos homens. A variabilidade da largura do passo aumenta com a idade e maior nas mulheres.

Neptune et al. (2004) e Liu et al. (2006) analisaram as contribuições dos grupos musculares para suporte e progressão do corpo durante a marcha. Os autores concluíram que os músculos flexores plantares dos tornozelos são a primeira fonte de aceleração para a frente durante o apoio final e constituem o grupo muscular chave para gerar suporte e progressão. O músculo gastrocnêmio age mais no aumento da velocidade de marcha do que o músculo solear. Os músculos extensores do quadril e joelho (glúteo máximo e vastos) dão suporte no apoio inicial e auxiliam na progressão. 0 glúteo médio contribui para suporte no médio apoio. Os dorsiflexores ajudam no processo de transferência do peso. A perda de força nesses músculos pode contribuir para a diminuição do passo, da passada e da velocidade da marcha.

Segundo Shkruratova et al. (2004) e Castro e col. (2000), pessoas idosas não aumentam a velocidade de marcha e o comprimento dos passos como o adulto jovem, possivelmente, para manter a estabilidade. A velocidade é considerada o melhor parâmetro isolado representativo do desempenho da marcha.

O objetivo do estudo de Shkruratova et al. (2004) foi verificar se idosos adaptam seus passos de maneira diferente dos jovens em resposta a várias perturbações do equilíbrio durante a locomoção. Os idosos apresentaram redução na velocidade, no comprimento da passada e maior 
tempo de duplo apoio, em comparação com os jovens. Isto geralmente é interpretado como adaptações relacionadas à idade para produzir uma marcha mais segura e menos desestabilizada, e compensar o aumento da aceleração do corpo durante a caminhada rápida.

Owings \& Grabiner (2004) avaliaram a influência da idade na variabilidade dos passos, velocidade de caminhada e o uso das barras de apoio durante marcha na esteira. Participaram 12 idosos com média de 73,4 anos e 8 jovens com média de 27,7 anos. Os idosos tiveram maior variabilidade de largura do passo em comparação com os jovens. A variabilidade do passo não foi afetada pela velocidade. A variabilidade do comprimento e a largura dos passos são maiores sem apoio das mãos do que com apoio.

A avaliação da marcha é essencial no monitoramento de recuperação ou declínio da função motora. O aumento da velocidade diminui o tempo de duração do passo, com aumento da cadência (passos mais curtos) nos indivíduos idosos (Zijlstra, 2004).

Henriksson \& Hirschfeld (2005), compararam estratégias empregadas por idosos e jovens saudáveis, durante o início da marcha, instruídos a atravessar a rua dentro do laboratório, quando o semáforo mudava de vermelho para verde. Os idosos assumiram uma posição de início irregular para facilitar o movimento para a frente, reduzindo a carga no balanço da perna, com um atraso na ativação dos músculos do tornozelo (tibial anterior). Os idosos, para melhorar a estabilidade, anteriorizam o centro de gravidade dentro da base de suporte e, iniciam a marcha com inclinação do tronco para 
a frente com passos mais curtos. O tempo de reação dos idosos aumenta sucessivamente durante os quatro experimentos repetidos e a atividade antecipatória do tibial anterior melhora, indicando a memorização do procedimento motor.

Lee et al. (2005) referem que a diminuição da extensão de quadril e aumento da inclinação anterior da pelve em idosos são fenômenos dinâmicos da marcha e não ocorrem durante a postura ortostática. Na caminhada auto-selecionada e na rápida, o idoso apresentou redução da extensão de quadril e aumento na inclinação anterior na pelve, com menor comprimento dos passos e velocidade de caminhada, em comparação com os jovens.

Barak et al. (2006) ivestigaram a cinemática da marcha, de 21 pessoas idosas que sofreram pelo menos uma queda há seis meses comparadas com idosos que nunca caíram, através de uma caminhada em velocidade auto-selecionada, em uma esteira. Os dois grupos mostraram aumento de cadência com o aumento da velocidade. $\mathrm{O}$ grupo com histórico de quedas mostrou passos mais curtos, cadência maior e diminuição da flexão plantar e extensão de quadril durante o final do apoio e aumento da flexão de quadril na fase de balanço.

O decréscimo na força se associa com a tendência a dar passos múltiplos para recuperar o equilíbrio nos idosos. A perda de força dos músculos abdutores e flexores do quadril e extensores de joelhos contribui para a menor estabilidade durante a fase de balanço e início do apoio (Maki \& Mclroy, 2006). 


\subsection{Efeito dos exercícios na marcha}

A prática de exercícios resistidos por indivíduos muito idosos (até 96 anos) aumentou a força muscular, velocidade de marcha e mobilidade funcional. A força muscular foi testada com o movimento de levantar da cadeira sem o uso das mãos e com a velocidade da marcha em seis metros de caminhada (Fiatarone et al.,1990).

Leiper \& Craik (1991) avaliaram a relação entre atividade física e velocidade de caminhada, em mulheres entre 64 e 94 anos. 25 mulheres eram sedentárias, 27 eram ativas e 29 faziam exercícios de forma intensa. As mulheres que faziam exercícios mais intensos caminhavam mais do que as sedentárias e foram capazes de andar mais devagar do que as outras pelo melhor controle motor e equilíbrio. A idade é o determinante primário da velocidade de caminhada e é mais importante que a atividade física e a massa peso corporal. Os autores sugerem a padronização da idade para se avaliar o desempenho de um grupo.

Judge et al. (1993) avaliaram o efeito de um programa de 12 semanas de exercícios resistidos e treinamento de equilíbrio na força muscular e marcha de 31 idosos com mais de 75 anos. Os autores verificaram aumento de força e da velocidade de marcha. Este estudo mostra que a melhora da força muscular aumenta a velocidade de marcha.

Ades et al. (1996) avaliaram o efeito de um programa de exercícios resistidos de 12 semanas, a marcha de uma amostra de 24 idosos saudáveis (65-79 anos). Os exercícios resistidos melhoraram a capacidade de marcha e a força dos membros inferiores. 
Sipila et al. (1996) avaliaram o efeito de um programa de 18 semanas de exercícios resistidos para flexão e extensão de joelhos, na força muscular e velocidade da caminhada em mulheres idosas. Houe melhora do desempenho muscular. As melhoras da marcha foram maiores quando os exercícios do joelho foram associados com fortalecimento dos flexores plantares.

Berg \& Lapp (1998) mostraram que exercícios resistidos com pesos ajustáveis no tornozelo, de baixa a moderada intensidade, realizados durante oito semanas, não tiveram efeito na estabilidade locomotora, na velocidade de marcha e no tempo de passo de um grupo de idosos.

Barret \& Smerdely (2002) compararam os efeitos de um programa de exercícios resistidos progressivos com um programa de exercícios de flexibilidade, mobilidade e qualidade de vida em um grupo de 44 idosos, com mais de 60 anos, realizado durante dez semanas. Os achados sugerem que o treinamento resistido é mais eficiente na melhora da força, marcha e equilíbrio do que os exercícios de flexibilidade.

Lamoureux et al. (2002) verificaram o efeito de um programa de exercício resistido aplicado por 24 semanas (três vezes por semana) na marcha, avaliada pela capacidade de dar passos sobre um obstáculo. Houve ganho de força (197 a 285\%) e mudanças específicas na cinética e cinemática da marcha: aumento do comprimento do passo, aumento da velocidade para atravessar um obstáculo e maior controle dos membros inferiores. 
Purser et al. (2003) avaliaram o efeito de um programa de nove meses de treinamento aeróbio progressivo em 134 idosos acima de 64 anos, de ambos gêneros. Os participantes foram divididos em dois grupos: 70 idosos treinaram por 40 minutos e 64, por 20 minutos. Foram avaliadas a força muscular dos membros inferiores e velocidade da marcha em dez metros de percurso. Houve associação linear entre força e velocidade. $O$ aumento de velocidade depende mais da variação de força durante o programa do que das condições musculares iniciais pré-treinamento.

Latham et al. (2004) realizaram uma revisão sistemática sobre o efeito do treinamento de força progressivo na redução da incapacidade física dos idosos. Os autores relataram que a fraqueza muscular está associada com diminuição da velocidade da marcha e maior risco de incapacidade e quedas nas pessoas idosas. Entretanto, a força pode ser aumentada nesses indivíduos, com exercícios resistidos progressivos, ainda que pessoas com limitação funcional preexistente tenham menor ganho de força.

Lopopolo et al. (2006) avaliaram, em revisão sistemática, o efeito do exercício terapêutico (tipo, intensidade e dose) na velocidade de marcha em idosos. O exercício resistido e a combinação de exercício aeróbio com outro tipo têm efeitos na velocidade da marcha auto-selecionada. Exercícios de alta intensidade e alta dosagem também têm efeito significativo na velocidade da marcha auto-selecionada. Nenhum exercício afeta a velocidade rápida da marcha. Exercícios de baixa a moderada intensidade ou baixa dosagem não apresentam efeitos na velocidade da marcha. 
Krebs et al. (2007) treinaram 50 idosos (62-85 anos), três a cinco vezes por semana, durante seis semanas em suas casas de duas formas: treinamento funcional pela execução das atividades da vida diária com marcha, subir degraus, sentar e levantar treinamento com exercícios resistidos progressivos com bandas elásticas e avaliarm o efeito do treinamento na marcha. O grupo funcional teve velocidade de marcha maior que do grupo exercícios resistidos, mas ambos melhoraram o desempenho. Os dados sugerem que para se obter melhora da marcha, o treinamento funcional foi mais vantajoso do que o exercício resistido com bandas elásticas.

Menz et al. (2007) analisaram a marcha por meio da aceleração da cabeça e pelve em 100 idosos (73-93 anos), enquanto caminhavam em um percurso de 20 metros de superfície irregular formada por várias camadas (espuma, borracha, madeira e grama artificial). Avaliaram a função sensóriomotora e o medo de cair. Os achados sugerem que o movimento da cabeça ao caminhar é um dos objetivos primários do sistema de controle postural. A função sensório-motora é uma variável independente que provém um indicativo da habilidade física geral do indivíduo. O medo de cair influencia na mobilidade e na marcha dos indivíduos, podendo resultar em um menor comprimento dos passos e maior cautela ao caminhar; além disso, a redução do medo é acompanhada de melhora na função sensório-motora. 


\section{OBJETIVOS}

\subsection{Objetivos gerais}

Avaliar a marcha em mulheres idosas na faixa etária de 65 a 74 anos, sedentárias e ativas e de acordo com o tipo de exercício físico praticado: exercícios resistidos (com equipamentos) e exercícios gerais (exercícios com predominância aeróbia, flexibilidade, calistênicos e resistidos de baixa intensidade).

\subsection{Objetivos específicos}

Comparar os grupos com relação aos seguintes parâmetros:

- $\quad$ velocidade da marcha;

- comprimento dos passos;

- comprimento da passada;

- largura do passo;

- cadência da marcha. 


\section{MÉTODO}

\subsection{Delineamento do estudo}

Estudo transversal e controlado.

\subsection{Casuística}

A casuística do estudo foi selecionada dentre 60 indivíduos do sexo feminino, saudáveis (sem queixas ou perdas funcionais no momento do teste), com idade média de 68,9 \pm 3,1 e IMC (índice de massa corporal) de $26,8 \pm 3,8$. Todas as mulheres participantes eram idosas, de acordo com a definição da OMS (Organização Mundial de Saúde), que considera idosa a pessoa com mais de 60 anos.

O contato foi feito por telefone e, após uma breve entrevista, foi feito o convite para participar da pesquisa. Foi agendada uma avaliação clínica individual para verificar o cumprimento dos critérios de inclusão. O período de avaliações se estendeu de abril a setembro de 2006. Os indivíduos incluídos assinaram o termo de consentimento livre e esclarecido, da Faculdade de Medicina da Universidade de São Paulo, devidamente autorizado pela CAPPesq (Anexo I).

No estudo foram incluídas 20 mulheres idosas sedentárias (pelo menos doze meses sem nenhum tipo de exercício físico), 20 mulheres idosas ativas que faziam exercícios resistidos (musculação) no CECAFI Centro de Estudos em Ciências da Atividade Física da Disciplina de Geriatria da Faculdade de Medicina da Universidade de São Paulo, e 20 mulheres idosas ativas que faziam exercícios gerais praticantes de exercícios com predominância aeróbia, flexibilidade, calistênicos e resistidos de baixa 
intensidade) no Conjunto Desportivo Baby Barioni, da Secretaria da Juventude, Esporte e Lazer do Estado de São Paulo. O protocolo de pesquisa número 405/05 foi aprovado pela CAPPesq em 24 de novembro de 2005 (Anexo II).

\subsubsection{Critérios de inclusão}

a) Idosas saudáveis e independentes, sem limitação nas atividades da vida diária.

b) Idosas que conseguiam caminhar de maneira independente em ambientes externos pelo menos um quarteirão.

c) Idosas que conseguiam caminhar por quatro metros de acordo com a pontuação máxima do teste de velocidade de caminhada (Guralnick et al., 2000).

d) Ausência de alterações degenerativas que comprometessem a marcha, de acordo com o critério anterior.

e) Ausência de lesões, traumas, fraturas ou cirurgias de membros inferiores pelo menos três anos antes do estudo ou que impedissem a marcha.

f) Ausência de doenças incapacitantes que limitassem a permanência em posição ortostática e a marcha.

g) Índice de massa corporal $<33$.

h) Ausência de problemas visuais que impedissem a locomoção independente.

i) Sem hipotensão postural e alterações do sistema vestibular no momento do teste. 
j) Grupo Sedentários - não praticante de exercícios ou atividades controladas por doze meses ou mais (ACSM, 1998).

k) Grupo Gerais - realizava atividades aeróbias três a cinco vezes por semana, 20 a 60 minutos contínuos, há doze meses ou mais.

I) Grupo Resistidos - realizava treinamento resistido duas vezes por semana, fazendo de seis exercícios para grupos musculares maiores, há doze meses ou mais.

m) Aceitar participar do projeto e assinar o termo de consentimento.

\subsubsection{Descrição da casuística}

$\mathrm{Na}$ Tabela 1 constam os dados dos indivíduos avaliados por grupo:

Grupo Gerais (praticante de exercícios com predominância aeróbia, flexibilidade, calistênicos e resistidos de baixa intensidade), Grupo Resistidos (exercícios resistidos) e Grupo Sedentários (não praticante de exercícios físicos).

Tabela 1 - Dados antropométricos (média e desvio padrão) dos indivíduos avaliados por grupo.

\begin{tabular}{|c|c|c|c|}
\hline VARIÁVEL & $\begin{array}{l}\text { GRUPO } \\
\text { GERAIS * }\end{array}$ & $\begin{array}{l}\text { GRUPO } \\
\text { RESISTIDOS* }\end{array}$ & $\begin{array}{l}\text { GRUPO } \\
\text { SEDENTÁRIOS * }\end{array}$ \\
\hline ldade $^{\star *}$ (anos) & $68,6 \pm 3,3$ & $69 \pm 2,9$ & $69,2 \pm 3,4$ \\
\hline Peso (kg) & $66,1 \pm 10,5$ & $62,7 \pm 8,5$ & $62,8 \pm 10,1$ \\
\hline Altura (cm) & $154,9 \pm 4,7$ & $154,2 \pm 6,0$ & $154 \pm 6,8$ \\
\hline $\mathrm{IMC}\left(\mathrm{kg} / \mathrm{cm}^{2}\right)$ & $27,5 \pm 4,2$ & $26,4 \pm 3,2$ & $26,5 \pm 4,0$ \\
\hline
\end{tabular}




\subsection{Metodologia de treinamento}

Após assinatura do termo de consentimento informado, todos os indivíduos foram submetidos a um questionário (Anexo III): identificação, idade, antecedentes pessoais e familiares, queixas clínicas e dolorosas, uso de medicamentos (informados pelo indivíduo ou familiar), dados antropométricos: peso e altura e tipo de atividade física praticada.

\subsubsection{Descrição dos grupos}

Os participantes selecionados foram separados em três grupos de acordo, com o exercício físico:

- Grupo Gerais - idosos saudáveis ativos, praticantes de exercícios com predominância aeróbia, flexibilidade, calistênicos e resistidos de baixa intensidade. As idosas realizavam duas vezes por semana uma variedade de exercícios em uma mesma sessão de uma hora, iniciada com uma caminhada de 20 minutos continuava com exercícios calistênicos para membros superiores e inferiores, exercícios resistidos de baixa intensidade com halteres, caneleiras ou o peso do próprio corpo e terminava com exercícios de alongamento e relaxamento. Além desses exercícios, uma a três vezes por semana faziam caminhadas, ou hidroginástica, ou natação por no mínimo 30 minutos.

- Grupo Resistidos - idosos saudáveis ativos, praticantes de exercícios resistidos por no mínimo um ano, duas vezes por semana, com duração de uma hora por sessão. Foram usados equipamentos da Biodelta/Linha 
Maxiflex para os seguintes exercícios: Press Peitoral, Remada, Extensão lombar, Leg Press, Flexões plantares e Flexões abdominais. Os músculos exercitados são: peitoral maior e menor, dorsais e escapulares, extensores da coluna lombar, extensores do joelho, glúteo máximo, gastrocnêmio, solear e abdominais. Foram realizadas três séries de oito a doze repetições, com intervalo de um a dois minutos entre as séries. Para aquecimento, na primeira série foi utilizado $50 \%$ do peso da terceira e na segunda série, $75 \%$. A carga da terceira série foi definida por aproximação sucessiva, sendo adequada para a realização de oito a doze repetições com dificuldade (escala de Borg 15). As cargas das três séries foram reajustadas sempre que diminuísse a dificuldade para a realização da terceira série. O esforço máximo, caracterizado por contração concêntrica lenta com respiração bloqueada, não foi utilizado.

- Grupo Sedentários - idosos saudáveis, inativos por no mínimo um ano.

As avaliações foram realizadas no Laboratório de Estudos do Movimento do Instituto de Ortopedia e Traumatologia do Hospital das Clínicas da Faculdade de Medicina da Universidade de São Paulo. O mesmo examinador realizou todos os procedimentos. Os indivíduos caminharam com sapatos padronizados tipo moleca com a numeração adequada, vestindo roupa confortável, em um corredor de 10 metros de comprimento por 60 centímetros de largura. 


\subsubsection{Avaliações}

\subsubsection{Avaliação da massa corporal e altura}

A medida da massa corporal e da altura do indivíduo foi feita com a utilização de uma balança antropométrica digital marca Filizola, com variação de zero a $150 \mathrm{~kg}$ e altura do estadiômetro de $97 \mathrm{~cm}$ a dois metros.

\subsubsection{Avaliação do tempo e da velocidade da marcha}

Foi solicitado aos indivíduos que caminhassem com passos habituais na velocidade auto-selecionada. Foram colocadas três cadeiras no início, no meio e no final da passarela para sentarem, caso fosse necessário. Os indivíduos caminhavam ao longo do percurso, seguidos de perto pelo avaliador, para dar-Ihes segurança, sem interferir na velocidade.

Os indivíduos caminharam sobre uma passadeira de carpete (tipo forração) de 10 metros de comprimento por 60 centímetros de largura. Foi

solicitado a cada indivíduo que caminhasse no seu passo habitual e confortável. Após um período de adaptação (duas voltas), foi medido o tempo gasto em segundos para percorrer, em passos habituais, as distâncias predeterminadas de 4 e 10 metros. Foi excluído um metro para as fases de aceleração e de desaceleração, que foram feitas fora da passadeira (Figura 1).

Para o início do teste, era dado o sinal "pode ir", sendo que o cronômetro iniciava-se após a aceleração. Foram realizadas três medidas, 
ou seja, o teste foi repetido três vezes, e foi utilizado o tempo médio das três medidas. Esta medida foi realizada com o cronômetro Digital Sports stop watch HS-6 (Casio). Para o cálculo da velocidade de caminhada em m/s, considerou-se a distância total do percurso fixo, em 4 metros e 10 metros, dividida pelo tempo gasto para vencer a distância (Judge et al., 1993; Sipila et al., 1997; Guralnick et al., 2000; Ostir et al., 2002; Purser et al., 2003; Dickstein et al., 2005).

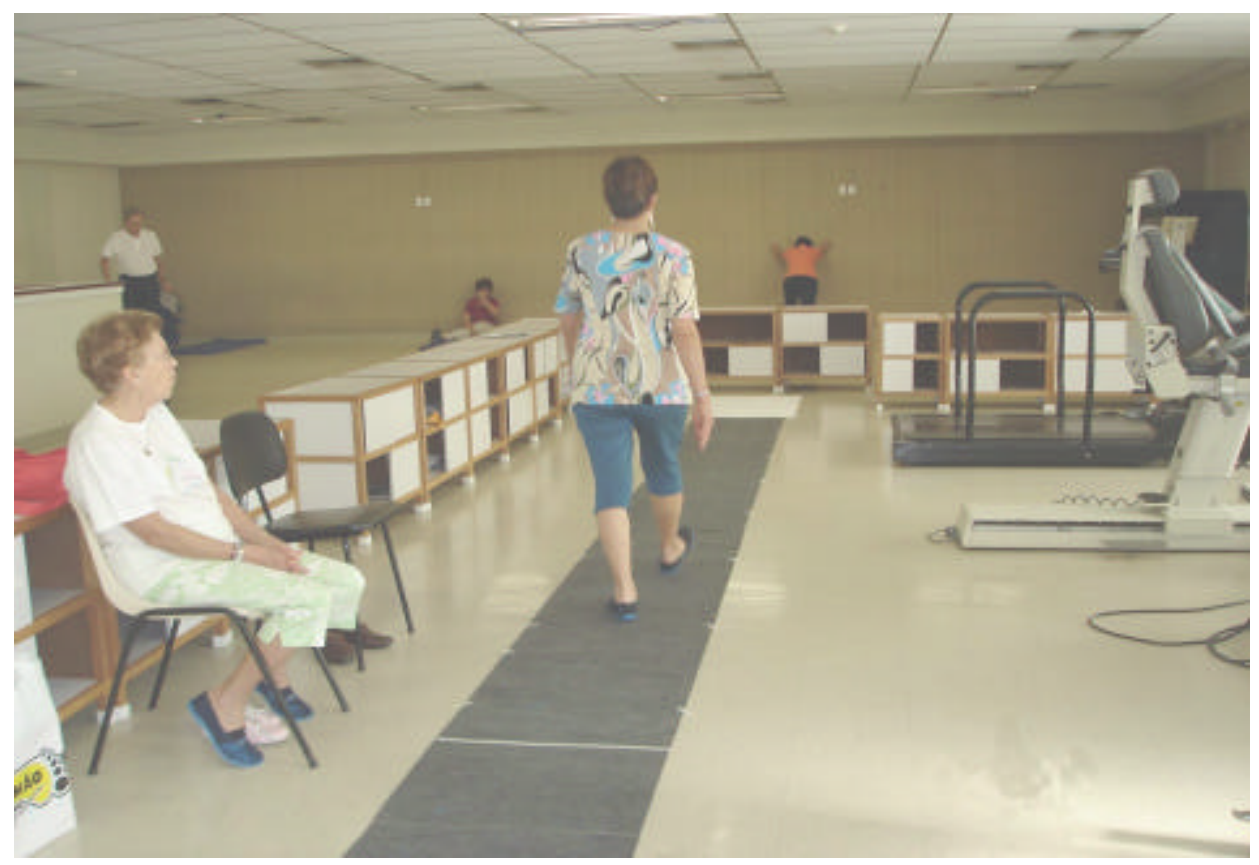

Figura 1. Medida do tempo (segundos) e da velocidade de caminhada $(\mathrm{m} / \mathrm{s})$ em 4 e 10 metros.

\subsubsection{Avaliação do comprimento do passo e da passada e largura do passo}

Os indivíduos caminharam em formulários contínuos de papel branco de 10 metros de comprimento por 70 centímetros de largura, estendidos sobre a passadeira, em velocidade auto-selecionada e habitual, em uma única tomada. Todos os indivíduos caminharam previamente sobre a passadeira para se familiarizar com o teste e com o calçado. A sola do 
calçado foi marcada com tinta azul e foi-lhes pedido que caminhassem com os passos habituais, deixando pegadas na passadeira (Figura 2). 0 indivíduo partia da posição sentada, passava os dois pés na caixa de plástico com tinta azul, e ao sinal "pode ir", com a ajuda do avaliador, iniciava a caminhada. Foi excluído um metro para as fases de aceleração e desaceleração fora da passadeira. A seguir, foi medido o ponto médio dos calcanhares (situado a 3 centímetros da borda do calcanhar) e traçado, com uma régua de 60 centímetros, um esquadro e uma trena de 3 metros, o comprimento de cada passo e de cada passada e a largura dos passos, conforme Figuras 3, 4, 5 e 6. Foram descontados os três primeiros e os três últimos passos do percurso de 10 metros e utilizados os passos e passadas centrais para serem medidos (Judge et al., 1993; Sekiya et al., 1997; Castro e col., 2000; Stolze et al., 2000; Helbostad \& Moe-Nilssen, 2003; Dickstein et al., 2005).

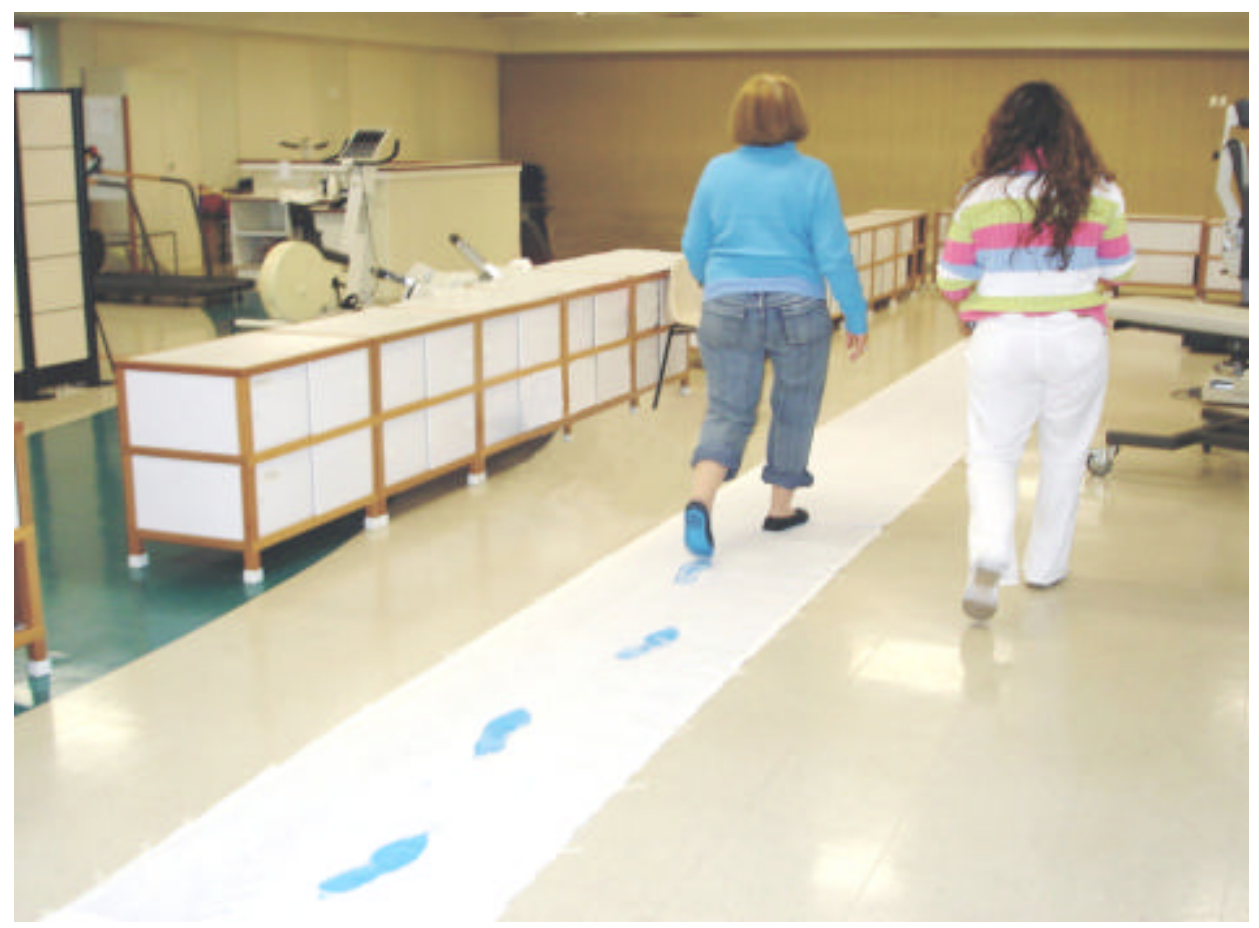

Figura 2. Pegadas com tinta azul em formulários contínuos de papéis brancos. 


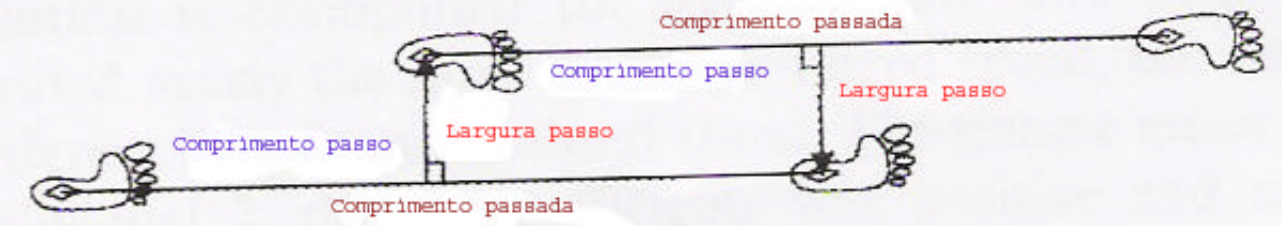

Figura3. Modelo utilizado para medição da largura e comprimento do passo e da passada (medido do centro do calcanhar) (Helbostad \& Moe-Nilssen, 2003).

As Figuras 4, 5 e 6 a seguir mostram o comprimento do passo, da passada e a largura do passo.

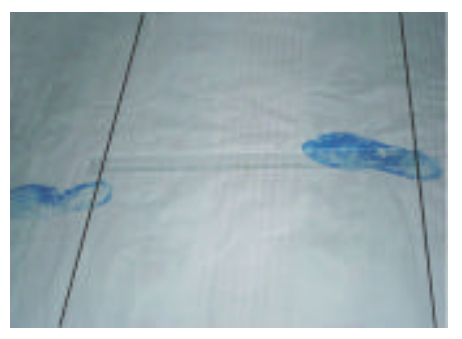

Figura 4. Comprimento do passo

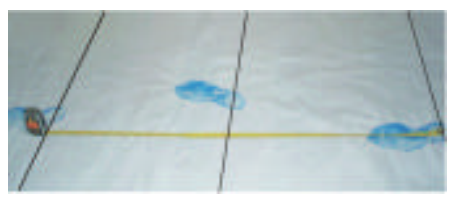

Figura 5. Comprimento da passada

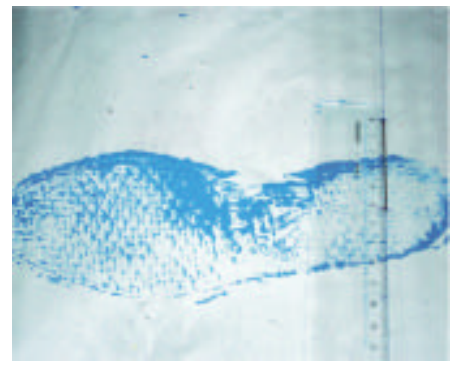

Figura 6. Largura do passo

\subsubsection{Avaliação da cadência}

A cadência, que é o número de passos por minuto, foi calculada por meio de uma regra de três. Contourse o número de passos marcados nos formulários contínuos (Figura 2) e dividiu-se pelo tempo gasto para percorrer 10 metros e no final multiplicou-se por 60 segundos (um minuto) (Judge et al., 1993; Castro e col., 2000; Stolze et al., 2000; Dickstein et al., 2005). 


\subsection{Análise estatística}

Neste estudo, foi realizada estatística descritiva, com cálculo da média e desvio padrão para todas as variáveis, sendo utilizado o programa Statistical Package for the Social Sciences (SPSS) versão 11.5 .1 for Windows.

As variáveis quantitativas analisadas (comprimento do passo e passada, largura do passo, cadência, altura e IMC) apresentaram distribuição normal. Para comparar as variáveis da marcha nos diferentes grupos, utilizoutse a análise de variância a um critério (ANOVA one way) e, para encontrar as diferenças estatísticas entre as médias, foi utilizado o teste posthoc LSD (least significant diference).

As variáveis quantitativas de velocidade com 4 metros, 10 metros e com tinta nos pés não apresentaram distribuição normal e foram logaritmizadas, após o qual se aplicou a análise de variância a um critério (ANOVA one way). A variável idade não apresentou distribuição normal e foi utilizado o teste Kruskal-Wallis.

O nível de significância adotado foi de $0,05(\mathrm{a}=5 \%$ e níveis descritivos (p) inferiores a este valor foram considerados significantes e representados por $\left(^{*}\right)$. 


\section{RESULTADOS}

Tabela 2. Média e desvio padrão do tempo de marcha (segundos) medido em 4 metros, 10 metros e 10 metros, com tinta nos pés, nos grupos Gerais, Resistidos e Sedentários.

\begin{tabular}{|c|c|c|c|}
\hline \multirow[t]{2}{*}{ VARIAVELL } & GRUPO & GRUPO & GRUPO \\
\hline & GERAIS & RESISTIDOS & SEDENTÁRIOS \\
\hline Tempo (4 m) & $3,23 \pm 0,22$ & $3,32 \pm 0,29$ & $3,87 \pm 0,39^{\star *}$ \\
\hline Tempo (10 m) & $8,19 \pm 0,62$ & $8,00 \pm 0,64$ & $9,74 \pm 0,98^{* *}$ \\
\hline $\begin{array}{llll}\text { Tempo } & \left(\begin{array}{lll}10 & \mathrm{~m} & \mathrm{c} /\end{array}\right. \\
\text { tinta) } & & & \end{array}$ & $8,52 \pm 0,93$ & $8,30 \pm 0,77$ & $10,76 \pm 1,71^{* *}$ \\
\hline
\end{tabular}

\section{ANOVA/ LSD $\left(p<0,001^{* *}\right)$}

Grupo Gerais < Grupo Sedentários - todas as variáveis $(p<0,001)^{* *}$

Grupo Resistidos < Grupo Sedentários - todas as variáveis $(p<0,001)^{\star *}$

Grupo Resistidos Grupo Gerais ( $p>0,05)$ NS

Tabela 3. Média e desvio padrão da velocidade da marcha (metros/segundo) em 4 metros, 10 metros e 10 metros, com tinta nos pés, nos grupos Gerais, Resistidos e Sedentários.

\begin{tabular}{llll}
\hline VARIÁVEL & $\begin{array}{l}\text { GRUPO } \\
\text { GERAIS }\end{array}$ & GRUPO RESISTIDOS & GRUPO SEDENTÁRIOS \\
\hline Velocidade $(4 \mathrm{~m})$ & $1,24 \pm 0,08$ & $1,21 \pm 0,10$ & $1,04 \pm 0,11^{\star *}$ \\
Velocidade (10 m) & $1,22 \pm 0,09$ & $1,25 \pm 0,10$ & $1,03 \pm 0,10^{* *}$ \\
$\begin{array}{l}\text { Velocidade }(10 \mathrm{~m} \mathrm{c/} \\
\text { tinta) }\end{array}$ & $1,18 \pm 0,13$ & $1,21 \pm 0,11$ & $0,95 \pm 0,16^{\star *}$ \\
\hline
\end{tabular}

ANOVA/ LSD $\left(p<0,001^{\star *}\right)$

Grupo Gerais > Grupo Sedentários - todas as variáveis $(p<0,001)^{\star *}$

Grupo Resistidos $>$ Grupo Sedentários - todas as variáveis $(p<0,001)^{\star \star}$

Grupo Resistidos Grupo Gerais ( $p>0,05)$ NS 
Há correlação nos três grupos, entre a velocidade da marcha entre os percursos de 4 metros e 10 metros $\left(r=0,82, p<0,001^{\star *}\right)$, entre o percurso de 4 metros e 10 metros com tinta $\left(r=0,76, p<0,001^{* *}\right)$ e entre o percurso de 10 metros e 10 metros com tinta $\left(r=0,89, p<0,001^{* \star}\right)$.

Há correlação significante por grupo entre tempo da marcha (segundos) e velocidade da marcha $(\mathrm{m} / \mathrm{s})$ :

na distância de 4 metros e 10 metros no grupo Gerais $(r=0,95$, $\left.p<0,001^{* *}\right)$ e no grupo Sedentários $\left(r=0,95, p<0,001^{* *}\right)$;

> na distância de 4 metros e 10 metros com tinta no grupo Gerais $\left(r=0,68, p<0,01^{*}\right)$ e no grupo Sedentários $(r=0,75, p<$ $\left.0,001^{* *}\right)$

na distância de 10 metros e 10 metros com tinta no grupo Gerais $\left(r=0,68, p<0,01^{*}\right)$, no grupo Sedentários $(r=0,75, p<$ $\left.0,001^{\star *}\right)$ e no grupo Resistidos $\left(r=0,81, p<0,001^{\star *}\right)$.

Não houve correlação entre tempo da marcha (segundos) e velocidade da marcha $(\mathrm{m} / \mathrm{s})$ :

na distância de 4 metros e 10 metros no grupo Resistidos ( $r=$ $0,11, p>0,05)$

na distância de 4 metros e dez metros com tinta no grupo Resistidos $(r=0,16, p>0,05)$. 
Tabela 4. Média e desvio padrão do comprimento dos passos e passadas (centímetros) medido em seis e em dez passos e passadas consecutivos nos grupos Gerais, Resistidos e Sedentários.

\begin{tabular}{llll}
\hline VARIAVEL & GRUPO GERAIS & GRUPO RESISTIDOS & GRUPO SEDENTARIOS \\
\hline $\begin{array}{l}\text { Comprimento } \\
\text { (seis passos) }\end{array}$ & $62,2 \pm 4,0$ & $61,0 \pm 4,9$ & $55,0 \pm 7,0^{\star \star}$ \\
$\begin{array}{l}\text { Comprimento } \\
\text { (dez passos) }\end{array}$ & $62,1 \pm 4,2$ & $60,1 \pm 4,8$ & $55,7 \pm 7,0^{\star *}$ \\
$\begin{array}{l}\text { Comprimento } \\
\text { (seis passadas) }\end{array}$ & $123,8 \pm 8,0$ & $120,1 \pm 9,9$ & $110,9 \pm 14,16^{\star \star}$ \\
$\begin{array}{l}\text { Comprimento } \\
\text { (dez passadas) }\end{array}$ & $124,5 \pm 8,3$ & $121,5 \pm 9,8$ & $111,2 \pm 14,17^{\star \star}$ \\
\hline
\end{tabular}

$$
\text { ANOVA/ LSD }\left(p<0,001^{* *}\right)
$$

Grupo Gerais $>$ Grupo Sedentários - todas as variáveis $(p<0,001)^{\star *}$

Grupo Resistidos $>$ Grupo Sedentários - todas as variáveis $(p<0,001)^{* *}$ Grupo Resistidos Grupo Gerais ( $p>0,05)$ NS

Há correlação entre o comprimento de seis passos e dez passos ( $r=$ $\left.0,97, p<0,001^{* *}\right)$ e entre 0 comprimento de seis passadas e dez passadas $\left(r=0,99, p<0,001^{\star \star}\right)$.

Há correlação significante nos três grupos (Gerais, Resistidos e Sedentários) entre altura dos indivíduos (metros) e comprimento dos passos e passadas (centímetros):

no comprimento de seis passos e altura $\left(r=0,39, p<0,01^{*}\right)$;

$>$ no comprimento de dez passos e altura $\left(r=0,44, p<0,001^{* \star}\right)$;

$>$ no comprimento de seis passadas e altura $(r=0,44, p<$ $\left.0,001^{* *}\right)$

$>$ no comprimento de dez passadas e altura $(r=0,43, p<$ $\left.0,001^{\star \star}\right)$. 
Tabela 5. Média e desvio padrão da largura do passo (centímetros) em seis e dez passos consecutivos nos grupos Gerais, Resistidos e Sedentários.

\begin{tabular}{lccc}
\hline VARIAVEL & GRUPO GERAIS & GRUPO RESISTIDOS & GRUPO SEDENTARIOS \\
\hline $\begin{array}{l}\text { Largura } \\
\text { (seis passos) }\end{array}$ & $6,92 \pm 1,79$ & $7,93 \pm 2,40$ & $6,98 \pm 2,71$ \\
$\begin{array}{l}\text { Largura } \\
\text { (dez passos) }\end{array}$ & $6,95 \pm 1,92$ & $8,05 \pm 2,23$ & $7,10 \pm 2,60$ \\
\hline
\end{tabular}

$$
\text { ANOVA/ LSD }(p>0,05) N S
$$

Grupo Gerais Grupo Resistidos Grupo Sedentários ( $>$ 0,05)NS

Tabela 6. Média e desvio padrão da cadência (número de passos/minuto) em 10 metros, com tinta nos pés, nos grupos Gerais, Resistidos e Sedentários.

\begin{tabular}{|c|c|c|c|}
\hline VARIAVEL & GRUPO GERAIS & GRUPO RESISTIDOS & GRUPO SEDENTARIOS \\
\hline Cadência & $109,23 \pm 13,61$ & $113,12 \pm 9,95$ & $97,92 \pm 11,13^{*}$ \\
\hline \multicolumn{4}{|c|}{ ANOVA/ LSD $\left({ }^{* *} p<0,05\right)$} \\
\hline \multicolumn{4}{|c|}{ Grupo Gerais $>$ Grupo Sedentários $(p<0,01)^{*}$} \\
\hline \multicolumn{4}{|c|}{ Grupo Resistidos > Grupo Sedentários $(p<0,001)^{\star *}$} \\
\hline \multicolumn{4}{|c|}{ Grupo Resistidos Grupo Gerais $(p>0,05)$ NS } \\
\hline
\end{tabular}

Há correlação significante entre a cadência e a velocidade de caminhada em 10 metros, com tinta, para os três grupos $(r=-0,71, p<$ $\left.0,001^{* \star}\right)$. A cadência foi correlacionada apenas no percurso de 10 metros, com tinta, porque somente nesta distância foi possível contar as pegadas em tinta. 


\section{DISCUSSÃO}

A dificuldade de locomoção é um dos fatores mais importantes de limitação funcional para os idosos. Vem sendo extensamente estudada e muitos são os autores que escrevem sobre o tema e que apontam para a relação entre as alterações da marcha e a presença de incapacidades nos idosos (Elble et al. 1991, Judge et al. 1993, Guralnick et al. 1995, Alexander 1996, Prince et al. 1997, Scarborough et al.1999, Daley e Spinks 2000, Brach et al. 2001, Hausdorff et al. 2001, Ostir et al. 2002, Lamoureux et al. 2003, Purser et al. 2003, Latham et al. 2004, Shkruratova et al. 2004, Graf et al. 2005 e Lopopolo et al. 2006). A avaliação da marcha é um importante elemento na propedêutica e avaliação funcional dos pacientes idosos. Existem vários métodos para realizar essa avaliação, porém seu custo é alto e muitos deles não têm utilidade na prática clínica diária. Nesta pesquisa foi escolhida uma avaliação simples, prática e de baixo custo, utilizando-se das medidas espaciais e temporais da marcha, variáveis que se modificam com a idade e a prática de exercício físico (Judge et al., 1993; Alexander, 1996; Bohannon et al., 1997; Stolze et al., 2000; Ostir et al., 2002; Reid et al., 2005; Dickstein et al., 2005).

A faixa etária dos indivíduos participantes desta pesquisa situourse entre 65 e 74 anos, de forma proposital, pois objetivava-se um grupo mais homogêneo, que permitisse uma melhor análise da prática e do tipo de exercício físico sobre as variáveis clínicas da marcha. Também se levou em conta, ao optar por essa faixa etária, a maior facilidade de controle sobre as 
doenças crônicas e comorbidades do que em grupos com mais idade, facilitando sua homegeneidade (Leiper \& Craik, 1991; Ostrosky et al., 1994).

Todos os indivíduos avaliados tinham o índice de Guralnick de quatro pontos, sendo esta a pontuação máxima, ou seja, todos caminhavam 4 metros em menos de 4,81 segundos (Guralnick et al., 2000). O maior tempo médio de caminhada (3,32 segundos) foi encontrado no Grupo Sedentários. Este foi um dos fatores de inclusão, mostrando que todos os indivíduos incluídos nesta amostra eram saudáveis e sem incapacidades para marcha independente.

A velocidade da marcha foi menor no Grupo Sedentários, com maior tempo de percurso em todas as distâncias avaliadas, em comparação com os Grupos Gerais e Resistidos. Estes grupos fizeram os percursos de 4 metros, 10 metros e 10 metros com tinta nos pés, em tempo menor que o Grupo Sedentários. Este resultado permite inferir que praticar exercícios de forma regular contribui para a manutenção da eficiência da marcha e que, possivelmente, perda de massa muscular e desuso podem ser responsáveis pela marcha menos eficiente. Muitos autores estudaram a velocidade da marcha, o comprimento e largura dos passos e passadas, a cadência e identificaram diferenças entre o adulto jovem e o idoso.

A redução da velocidade da marcha no idoso, em relação ao jovem, é consensual e ocorre, principalmente, em decorrência da diminuição do comprimento dos passos e passadas. O envelhecimento também aumenta a cadência (maior número de passos por minuto), fato que contribui para aumentar a estabilidade, porém aumenta o consumo de energia. Os idosos, 
no início da marcha, dão os primeiros passos mais curtos, para facilitar o movimento para a frente, com uma mudança mais gradual do centro de gravidade. Há também alargamento do passo, fato que contribui para aumentar o equilíbrio na marcha, mas pode facilitar as quedas. $O$ envelhecimento altera os parâmetros da marcha, fato que pode, no entanto, ser minimizado ou prevenido pela manutenção de um bom condicionamento físico (Elble et al., 1991; Oberg et al., 1993; Ostrosky et al., 1994; Judge et al., 1996; Kerrigan et al., 1998; Owings \& Grabiner, 2003 e 2004; Shkuratova et al., 2004; Henriksson \& Hirschfeld, 2005; Lee et al., 2005).

A perda de força muscular provoca diminuição do comprimento dos passos e passadas e da velocidade da marcha (Fiatarone et al., 1990; Leiper \& Craik., 1991; Elble et al., 1991; Judge et al., 1993; Oberg et al., 1993; Ostrosky et al., 1994; Ronsky et al., 1995; Alexander, 1996; Bohannon et al., 1997; Rubino, 2002; Henriksson \& Hirschfeld, 2005). Os resultados encontrados no presente estudo são coincidentes com estes autores, mostrando que os exercícios ajudam a manter uma marcha de boa qualidade, que não depende do tipo de exercício realizado.

A diminuição da velocidade da marcha é explicada pela diminuição da força e da massa muscular, mas também se constitui de uma das estratégias utilizadas para aumentar a estabilidade (Mcgibbon, 2003; Barak et al., 2006). Ainda, que não se tenha avaliado o equilíbrio por meio de testes específicos, não foram encontradas alterações de equiilíbrio durante os procedimentos de avaliação da marcha, fato que não causa surpresa pela casuística estudada. As alterações proprioceptivas do envelhecimento, 
relacionadas com a diminuição da velocidade de condução nervosa periférica, também podem estar implicadas na menor velocidade de marcha dos idosos, mas, possivelmente a perda da condição muscular tenha maior responsabilidade neste evento. A velocidade de marcha decresce com a idade e está relacionada com a diminuição do comprimento do passo. Judge et al. (1996) e Daley \& Spinks (2000) referem que o maior declínio da velocidade da marcha ocorre nas sétima e oitava décadas de vida e que este declínio varia de 12 a 16\%. Oberg et al. (1993) e Bohannon et al. (1997) relatam que a velocidade habitual de marcha decresce de 9 a11\%, entre 40 e 80 anos.

Buchner et al. (1996) afirmam que idosos frágeis (com pouca força) conseguem aumentar a velocidade de marcha quando ganham força muscular, mas depois que atingem força suficiente para caminhar, a velocidade não aumenta mais, somente se mantém. No presente estudo, o Grupo Sedentários apresentou a menor velocidade de marcha e menor força muscular, fato que pode ser explicado pela falta de força muscular de decorrente do próprio sedentarismo e que mostra concordância com Buchner et al. (1996). Outros autores, como Leiper \& Craik (1991), Judge et al. (1993), Grabiner et al. (2001), Barrett \& Smerdely (2002), Helbostad et al. (2003), Graf et al. (2005) e Menz et al. (2007), avaliaram indivíduos idosos em diferentes faixas etárias e mostraram diferenças significativas nos parâmetros temporais e espaciais da marcha.

O presente estudo avaliou indivíduos sedentários e ativos. Estes últimos foram separados em dois grupos específicos de exercícios e os 
resultados foram muito semelhantes aos de Leiper \& Craik (1991), que estudaram indivíduos idosos sedentários e ativos (diversos tipos de exercícios) e verificaram maior velocidade de caminhada no grupo que se exercitava, independente do tipo de exercício. Com relação, aos parâmetros temporais e espaciais da marcha, no presente estudo, os indivíduos ativos se diferenciaram dos sedentários, mas não entre si. Este achado permite inferir que a prática de atividade física melhora a qualidade da marcha, independente do tipo de exercício praticado. A especificidade do treinamento, que pode ser observada no Grupo Gerais, que se exercitava caminhando, não se mostrou importante nos resultados observados.

A maioria dos estudos que avaliou indivíduos idosos que praticavam exercícios resistidos verificou aumento da velocidade de caminhada habitual e da força muscular após dez ou doze semanas de treinamento (Judge et al., 1993; Ronsky et al., 1995; Ades et al., 1996; Barret \& Smerdely, 2002; Latham et al., 2004). Sipila et al. (1996) mostraram que o aumento da força muscular promove aumento da velocidade da marcha após dezoito semanas de exercícios resistidos. Lamoureux et al. (2002) verificaram melhora na força e na velocidade para vencer obstáculos após 24 semanas de treinamento. A variação de tempo para se obter ganhos funcionais depende do tipo e cargas de treinamento, fato que não foi importante no presente estudo, pois os dois grupos avaliados treinavam há pelo menos um ano. Berg \& Lapp (1998) encontraram pequena melhora na força, mas não na velocidade de marcha, com a prática de exercícios com pesos ajustáveis presos no tornozelo, realizados durante oito semanas. A falta de melhora na 
velocidade da marcha pode estar relacionada com a carga, limitando o ganho de força, e o tempo de treinamento. Fiatarone et al. (1990), no entanto, encontraram aumento da força e da velocidade da marcha dos idosos com oito semanas de exercícios resistidos em máquinas de musculação. Os exercícios aeróbios progressivos, feitos durante nove semanas, segundo Purser et al. (2003), promoveram aumento da velocidade da marcha. Os autores referem que a principal causa de melhora é a variação do ganho de força muscular e que o grau de fraqueza inicial não é importante, mostrando que mesmo idosos frágeis podem e devem praticar atividades físicas.

Lopopolo et al. (2006) fizeram uma revisão sistemática com metaanálise do tema e mostraram que os exercícios resistidos, sozinhos, podem melhorar a velocidade da marcha, assim como os exercícios aeróbios, desde que, estes últimos, sejam combinados com outro tipo de exercício.

Os resultados encontrados nos estudos similares da literatura e principalmente na revisão sistemática de Lopopolo et al. (2006) se aproximam, de forma muito consistente, dos resultados obtidos neste estudo. Relacionado ao presente estudo, observou-se que tanto nos indivíduos do grupo Gerais (programa de exercícios aeróbios, de flexibilidade, calistênicos e resistidos de baixa intensidade) como nos do grupo Resistidos (musculação em equipamentos) houve maior velocidade da marcha, quando comparados com o grupo Sedentários, mostrando que não importa o tipo, mas sim a prática de exercício. O grupo denominado Gerais fez um trabalho físico moderado, trabalhando várias aptidões físicas, 
inclusive a força e equilíbrio, mostrando que talvez um dos principais componentes que se relacionam com a melhora da marcha seja o ganho de força muscular.

A importância da especificidade do exercício físico para desenvolver determinadas aptidões físicas tem sido destacada em todos os programas de treinamento, e neste estudo, em particular, nos dois grupos de treinamento avaliados, essa regra foi observada. No grupo Gerais, a marcha foi um dos instrumentos do treinamento, na forma de caminhadas, e no grupo Resistidos os músculos antigravitacionais e que executam os movimentos da marcha foram fortalecidos de forma preferencial. Resta a dúvida se em outros tipos de exercícios, não gravitacionais e específicos, nos quais a força muscular é menos exigida, como exercícios aquáticos, de flexibilidade e de equilíbrio, haveria as mesmas repercussões sobre os parâmetros da marcha.

A qualidade das medidas foi avaliada no teste de 4 e 10 metros e nos 10 metros, com tinta nos pés. Os dois testes de 10 metros mostraram resultados correlatos entre si, em todos os grupos. O uso de um sistema de marcação com tinta nos pés foi seguro, não teve interferência nos parâmetros medidos (tempo e velocidade) e não causou instabilidades durante a execução do percurso. Na velocidade de 4 metros, no entanto, os grupos se comportaram de forma distinta: houve correlação entre o tempo e a velocidade nos grupos Sedentários e Gerais. O grupo Resistido apresentou melhor desempenho em 10 metros, embora não significante estatisticamente em relação ao grupo Geral. Este achado permite inferir que 
o teste com percurso de 10 metros é mais fácil de reproduzir e seria mais adequado para a avaliação dos parâmetros da marcha. Henriksson \& Hirschfeld (2005) mostraram que os indivíduos idosos têm um início de marcha irregular, que facilita o movimento para a frente e aumenta a estabilidade, o que está de acordo com nosso estudo. O treinamento específico melhora o desempenho inicial, o que também poderia explicar a marcha mais homogênea no grupo Gerais, nas três distâncias testadas.

A diminuição da força muscular dos membros inferiores se associa com as alterações dos passos, das passadas e da velocidade, como foi observado no presente estudo, em que os indivíduos idosos ativos (grupo Gerais e Resistidos) mostraram passos e passadas mais longos e maior velocidade que o grupo Sedentários. A diminuição do comprimento dos passos é um dos fatores responsáveis pela diminuição da velocidade da marcha (Oberg et al., 1993; Ostrosky et al., 1994; Judge et al., 1993 e 1996; Prince et al., 1997; Kerrigan et al., 1998; Scarborrough et al., 1999; Stolze et al., 2000; Riley et al., 2001; Hausdorff et al., 2001; Zijlstra, 2004), mas o aumento da idade e o sexo também afetam a marcha normal.

A diminuição do comprimento da passada, nos idosos, está relacionada com a fraqueza dos extensores do quadril e dos flexores plantares do tornozelo, no final da fase de apoio. A diminuição da velocidade pode estar relacionada com o aumento da flexão do quadril e a redução da habilidade para propulsão anterior do corpo. A prática regular do exercício físico preserva os fatores determinantes da marcha, pela manutenção da força muscular, flexibilidade e equilíbrio, o que retarda ou impede as perdas 
funcionais. Este fato justifica os achados do presente estudo (Ronsky et al., 1995; Judge et al., 1996; Kerrigan et al., 1998; Castro et al., 2000; Mcgibbon et al., 2003; Riley et al., 2001; Rubino, 2002; Shkruratova et al., 2004; Lee et al., 2005; Barak et al., 2006; Krebs et al., 2007).

A perda de força muscular dos membros inferiores relacionada à idade pode contribuir para aumentar a instabilidade durante a fase de balanço e apoio inicial, diminuindo a velocidade e o comprimento dos passos e das passadas. Elble et al. (1991), Oberg et al. (1993), Ostrosky et al. (1994), Judge et al. (1996), Scarborough et al. (1999), Neptune et al. (2004) e Liu et al. (2006) relataram que a perda de força do quadríceps diminui o comprimento dos passos e a velocidade da marcha. Esta diminuição pode ser explicada pela falta de ação do músculo reto femoral, que age durante a fase final do apoio, inicialmente como flexor do quadril, depois como extensor do joelho, acelerando a perna até o início do balanço, que é um dos determinantes do comprimento do passo e da velocidade. A ação do quadríceps durante a marcha gera um maior momento para a frente e contribui para a manutenção e o aumento da velocidade. Segundo os mesmos autores, é de suma importância a manutenção da força do quadríceps para a estabilidade dinâmica da marcha, para o comprimento da passada e a velocidade da marcha.

O prejuízo primário do equilíbrio, causado pelo envelhecimento biológico e multifatorial, leva ao medo de cair e à perda de força muscular, também agravada pela inatividade. Os indivíduos ficam mais cansados durante as atividades, dentre elas, a marcha, com conseqüente diminuição 
de velocidade. A perda da condição física leva a maior inatividade, gerando um ciclo vicioso, que faz aumentar a incapacidade funcional.

A análise das variáveis espaciais foi feita pela contagem do número de passos e passadas, avaliando-se se os passos seriam suficientes e adequados, garantindo a confiabilidade do teste. A correlação entre as médias de seis passos e dez passos foi boa, mostrando que poucos passos são suficientes para uma análise, e foi possível afirmar que os indivíduos caminharam da mesma forma durante todo o teste.

Daley \& Spinks (2000) referem que o comprimento da passada, nos jovens, varia de 151 a $170 \mathrm{~cm}$ e, nos idosos, de 135 a $153 \mathrm{~cm}$. O trabalho não faz referência, no entanto, à prática de exercício físico e à altura dos indivíduos testados, sendo difícil a comparação direta com este estudo. Richard (1995) afirma que a variável altura do indivíduo deve ser avaliada, mesmo em indivíduos da mesma idade e peso, pois ela pode afetar o comprimento dos passos. No presente estudo, as alturas foram pareadas, o que nos permite inferir que as diferenças encontradas no comprimento das passadas entre os grupos podem estar relacionadas à prática de exercício físico. O comprimento das passadas variou de 111,2 a 142,1 cm (grupo Gerais), de 101 a 140,7 cm (grupo Resistidos) e de 86,8 a 137,7 cm (grupo Sedentários). Os valores encontrados são menores quando comparados com os dados de Daley \& Spinks (2000), mas esta variação pode ser explicada pela altura, que não foi citada.

Castro e col. (2000) fizeram um estudo muito similar a este, avaliando a velocidade da marcha e o comprimento do passo. Encontraram, em 
comparação ao presente estudo, valores maiores que os do grupo Sedentários e menores que os dos grupos Gerais e Resistidos. Pode-se inferir que, nestes dois últimos grupos, a melhor condição muscular foi determinante para o aumento da velocidade e do tamanho dos passos.

Oberg et al. (1993) fizeram a mesma avaliação, mas não levou em conta a altura dos indivíduos. Os valores encontrados são maiores que os do grupo Sedentários e menores que os dos grupos Gerais e Resistidos do presente estudo, apontando, de novo, para a condição muscular como determinante da maior velocidade e comprimento do passo.

Quadro 1. Descrição dos resultados de autores diferentes e dos três grupos presentes no estudo com 60 a 69 anos.

\begin{tabular}{|l|c|c|c|c|c|}
\hline & $\begin{array}{c}\text { OBERG } \\
(1993)\end{array}$ & $\begin{array}{c}\text { CASTRO } \\
(2000)\end{array}$ & GERAIS & RESISTIDOS & SEDENTÁRIOS \\
\hline $\begin{array}{l}\text { Velocidade } \\
(\mathrm{m} / \mathrm{s})\end{array}$ & 1,15 & 1,05 & 1,22 & 1,25 & 1,03 \\
\hline Altura $(\mathrm{cm})$ & - & 159 & 154,9 & 154,2 & 154 \\
\hline $\begin{array}{l}\text { Comprimento } \\
\text { Passos }(\mathrm{cm})\end{array}$ & 55,3 & 52,1 & 62,2 & 61,0 & 55,0 \\
\hline
\end{tabular}

O Quadro 1 resume os achados de trabalhos similares a este e mostra de forma clara o papel dos exercícios e da condição muscular na manutenção dos parâmetros da marcha: comprimento do passo e velocidade da marcha.

Ainda que a força muscular e a amplitude de movimento dos membros inferiores não tenham sido medidas de forma direta, a maior velocidade e comprimento do passo e da passada dos indivíduos ativos 
(Grupos Gerais e Resistidos) podem estar relacionados com indivíduos mais fortes, mais flexíveis e mais aptos para as atividades diárias, pois tiveram melhor desempenho nos testes de marcha.

A largura do passo não foi diferente entre os grupos, o que seria esperado, pois o posicionamento médio-lateral dos pés é mais dependente de fatores antropométricos (largura da bacia, rotação, anteversão e retroversão da bacia). O aumento da base de sustentação e da largura dos passos pode ocorrer com a idade e com a perda da visão.

O posicionamento dos pés é um mecanismo de controle de equilíbrio proporcional à aceleração do centro de massa. Quanto mais devagar a pessoa caminha, maior o tempo de duplo apoio; os pés se posicionam mais longe da linha média e maiores são os desvios (Brach et al., 2001; Helbostad \& Moe-nilssen, 2003; Dickstein et al., 2005). Sekiya et al. (1997) referem maior variabilidade da largura do passo em velocidades rápidas, dependendo do equilíbrio. A variação da largura do passo com o envelhecimento não é consensual na literatura: Brach et al. (2001), Grabiner et al. (2001) e Helbostad \& Moe-nilssen (2003) referem que há modificações, porém Stolze et al. (2000) não verificaram essas medidas. Não foi encontrado trabalhos relacionando a largura do passo com o exercício físico e, no presente estudo, não se observou variação nesse parâmetro nos grupos pesquisados.

O comprimento da passada pode afetar a variabilidade espacial, assim como a cadência afeta a variabilidade temporal (Sekiya et al., 1997; Danion et al., 2003). Neste estudo, observourse a correlação da cadência 
com a velocidade nos 10 metros com tinta nos pés, mostrando que o teste foi bem executado e que os indivíduos caminharam da mesma maneira em todo o percurso.

O grupo Sedentários apresentou menor comprimento dos passos, velocidade de marcha e cadência. Estes achados são similares aos de Ostrosky et al. (1994), Alexander (1996), Sekiya et al. (1997), Riley et al. (2001) e Dickstein et al. (2005), mas discordantes dos resultados de Elble et al. (1991), Oberg et al. (1993), Judge et al. (1996), Prince et al. (1997), Kerrigan et al. (1998) e Lee et al. (2005). Estes últimos referem diminuição do comprimento do passo e velocidade, porém com a cadência mantida ou aumentada. Estes achados podem sugerir uma variação inter-testes, mas que também pode ser explicada por uma maior dificuldade na realização da fase de balanço, diminuindo muito o tamanho de cada passo. $\mathrm{O}$ indivíduo deve dar muitos passos, curtos e ineficientes, aumentando a cadência, mas não a velocidade. Maki \& Mclroy (2006) e Barak et al. (2006) mostraram essa tendência de dar múltiplos passos curtos para manter e recuperar o equilíbrio. Este fato aumenta o risco de quedas e está associado à perda de força muscular, com velocidade e comprimento de passos menores. Observou-se, nos grupos Gerais e Resistidos, que a cadência média (109 e 113 passos/minuto, respectivamente) esteve próxima dos valores normais para uma população adulta do mesmo sexo (110 a 115 passos/minuto). 0 grupo Sedentários teve média menor (98 passos/minuto) dentro do estudo e na comparação com outros trabalhos. Os idosos ativos tiveram cadência semelhante à dos indivíduos adultos jovens. 
Os indivíduos idosos caminham com passos mais curtos, tem fase de duplo apoio mais longa, movimentam menos a pelve e o tornozelo, têm redução da flexão plantar de tornozelo e da extensão de quadril no final da fase de apoio e compensam a fraqueza dos flexores plantares utilizando os músculos flexores do quadril na fase de balanço. As intervenções que melhorem a potência do tornozelo, joelho e quadril podem aumentar o comprimento do passo e a velocidade da marcha, mesmo nas pessoas idosas (Judge et al.,1996; Mcgibbon, 2003; Barak et al., 2006; Krebs et al., 2007). Os indivíduos idosos diminuem o comprimento do passo e da passada pela perda da flexibilidade, pela diminuição da força muscular dos membros inferiores ou pela deficiência do equilíbrio, e tentam compensar essas alterações aumentando a fase de duplo apoio. A avaliação da marcha nos idosos, portanto, deve ser abrangente, diversificada e multifatorial (força, equilíbrio, amplitudes de movimento, audição, visão, funções labirínticas e propriocepção) e deve avaliar os resultados das diferentes intervenções.

Este estudo mostrou de forma muito clara que o exercício físico regular melhora os parâmetros da marcha e ajuda na manutenção da funcionalidade e independência. Não conseguiu mostrar a superioridade de um tipo de atividade sobre outro, mas os caminhos apontam, de forma inequívoca, para o grande benefício dos exercícios para os idosos. Mostrou também que programas simples, exeqüíveis e de baixo custo promovem importantes ganhos funcionais na qualidade da marcha e devem contribuir para a prevenção de quedas, fraturas, incapacidades e óbitos. Ainda que os exercícios resistidos não tenham sido superiores aos exercícios gerais na 
avaliação da marcha, o treinamento da força muscular foi feito em ambos os programas (marcha e outros exercícios de carga baixa que trabalharam a força muscular), mostrando a importância desses exercícios para a manutenção do ortostatismo e marcha.

A sarcopenia e a perda de força muscular são elementos destacados na etiologia das incapacidades relacionadas com o envelhecimento e precisam ser combatidas de forma sistemática. As evidências apontam que indivíduos idosos melhoram sua força muscular com exercícios e que esta melhora se associa diretamente com a maior autonomia e qualidade de vida. 


\section{CONCLUSÕES}

A marcha apresentou parâmetros espaciais e temporais melhores nos grupos ativos (Gerais e Resistidos) do que no de sedentários.

Não houve diferença nos parâmetros espaciais e temporais da marcha entre os indivíduos que faziam exercícios resistidos ou exercícios gerais.

O exercício físico pode ter influenciado nos parâmetros espaciais da marcha do que a altura do indivíduo.

O parâmetro largura do passo é mais dependente dos fatores antropométricos do que da prática de atividade física. 


\section{CONSIDERAÇÕES FINAIS}

Os indivíduos utilizaram sapatos padronizados para que o tipo de calçado não interferisse na análise dos passos. Porém, o uso de calçado padronizado pode influenciar na marcha de indivíduos que não estão acostumados. A caminhada não foi realizada sem o uso de calçados para que seja o mais próximo da marcha normal.

É importante salientar o uso de uma tinta a base de água e não a base de óleo. Esta última apresenta uma melhor fixação das pegadas de tinta no formulário de papel, porém, faz com que os indivíduos possam vir a escorregar.

Este trabalho foi de relevância para mostrar a importância do exercício físico na marcha de idosos na faixa etária de 65 a 74 anos. Resta saber, em perspectivas futuras, se em indivíduos mais idosos ou nonagenários, também apresentariam esta influência. 


\section{REFERÊNCIAS BIBLIOGRÁFICAS}

ADES PA, BALLOR DL, ASHIKAGA T, UTTON JL, NAIR KS. Weight training improves walking endurance in healthy elderly persons. Annals Intern Med 1996;124(6):568-572.

ALEXANDER NB. Gait disorders in older adults. J Am Geriatr Soc 1996;44:434-451.

AMERICAN COLLEGE OF SPORTS MEDICINE. The recommended quantity and quality of exercise for developing and maintaining cardiorepiratory and muscular fitness, and flexibility in healthy adults. Med Sci Sports Exerc 1998;30:975-991.

BARAK Y, WAGENAAR RC, HOLT KG. Gait characteristics of elderly people with a history of falls: a dynamic approach. Phys Ther 2006;86 (11):15011510.

BARRET C, SMERDELY P. A comparison of community-based resistance exercise and flexibility exercise for seniors. Austr J Phys 2002;48:215-219.

BERG WP, LAPP BA. The effect of a practical resistance training intervention on mobility in independent, community- dwelling older adults. J Aging Phys Act 1998;6:18-35

BOHANNON RW. Comfortable and maximum walking speed of adults aged 20-79 years: reference values and determinants. Age Ageing 1997;26(1):1519.

BRACH JS, BERTHOLD R, CRAIK R, VANSWEARINGEN J, NEWMAN A. Gait variability in community-dwelling older adults. J Am Geriatr Soc 2001;49:1646-1650. 
BUCHNER DM, LARSON EB, WAGNER EH, KOEPSELL TD, LATEUR BJ. Evidence for a non-linear relationship between leg strength and gait speed. Age Ageing 1996;25:386-391.

CASTRO CLN, SANTOS JACB, LEIFELD OS, BIZZO LV, SILVA LC, ALMEIDA TF et al. Estudo da marcha em idosos - resultados preliminares. Acta Fisiátrica 2000;7(3):103-107.

CHAIMOWICZ F. A saúde dos idosos brasileiros às vésperas do século $X X I$ : problemas, projeções e alternativas. Rev Saúde Pública 1997;31(2):184-200.

CLARKE MSF. The effects of exercise on skeletal muscle in the aged. $J$ Musculoskel Neuron Interact 2004;4(2):175-178.

DALEY MJ, SPINKS WL. Exercise, mobility and aging. Sports Med 2000;29(1):1-12.

DICKSTEIN R, DUNSKY A, NADEAU S, ABULAFFIO N. Speed-dependent deviations from a straight-ahead path during forward locomotion in healthy individuals. Am J Phys Med Rehabil 2005;84: 330-337.

ELBLE RJ, THOMAS SS, HIGGINS C, COLLIVER J. Stride-dependent changes in gait of older people. J Neurol 1991;238:1-5.

FARINATTI PTV, LOPES LNC. Amplitude e cadência do passo e componentes da aptidão muscular em idosos: um estudo correlacional multivariado. Rev Bras Med Esp 2004;10(5):389-394.

FIATARONE MA, MARKS EC, RYAN ND, MEREDITH CN, LIPSITZ LA, EVANS WJ. High-intensity strength training in ronagenarians - Effects on skeletal muscle. JAMA 1990;263(22):3029-3034. 
FREITAS EV, PYL, NÉRI AL, CANÇADO FAX, GORZONI ML, ROCHA SM. Tratado de geriatria e gerontologia, Rio de Janeiro: Guanabara Koogan, 2002.

FRONTERA WR, MEREDITH CN, O'REILLY KP, KNUTTGEN HG, EVANS WJ. Strength conditioning in older men: skeletal muscle hypertrophy and improved function. J Appl Physiol 1998;64:1038- 1044.

FRONTERA WR, HUGHES VA, FIELDING RA, FIATARONE MA, EVANS WJ, ROUBENOFF R. Aging of skeletal muscle: a 12-yr longitudinal study. J Appl Physiol 2000;88:1321-1326.

FRONTERA WR. Exercício físico e reabilitação, Porto Alegre: Artmed, 2001.

GARRIDO R, MENEZES PR. O Brasil está envelhecendo: boas e más notícias por uma perspectiva epidemiológica. Rev Bras Psiquiatr 2002;24(supl I):3-6.

GRABINER PC, BISWAS ST, GARBINER MD. Age-related changes in spatial and temporal gait variables. Arch Phys Med Rehabil 2001; 82:31-35.

GRAF A, JUDGE JO, OUNPUU S, DARRYL GT. The effect of walking speed on lower-extremity joint powers among elderly adults who exhibit low physical performance. Arch Phys Med Rehabil 2005;86: 2177-2183.

GURALNICK JM, SIMONSICK EM, FERRUCIL, GLYNN RJ, BERKMAN LF, BLAZER DG et al. A short physical performance battery assessing lower extremity function: association with self-reported disability and prediction of mortality and nursing home admission. J Gerontol 1994;49(2):85-94. 
GURALNICK JM, FERRUCCI L, SIMONSICK EM, SALIVE ME, WALLACE RB. Lower-extremity function in persons over the age of 70 years as a predictor of subsequent disability. N Engl J Med 1995;332: 556-561.

GURALNICK JM, FERRUCCI L, PIEPER CF, LEVEILLE SG, MARKIDES KS, OSTIR GV et al. Lower extremity function and subsequent disability: Consistency across studies, predictive models, and value of gait speed alone compared with the short physical performance battery. J Gerontol 2000;55A(4):M221-231.

HAUSDORFF JM, NELSON ME, KALITON D, LAYNE JE, BERNSTEIN MJ, NUERNBERGER A et al. Etiology and modification of gait instability in older adults: a randomized controlled trial of exercise. $J$ Appl Physiol 2001;90:2117-2129.

HELBOSTAD $\perp$, MOE-NILSSEN R. The effect of gait speed on lateral balance control during walking in healthy elderly. Gait Posture 2003;18:2736.

HENRIKSSON M, HIRSCHFELD $H$. Physically active older adults display alterations in gait initiation. Gait Posture 2005;21:289-296.

HURLEY M, REES J, NEWHAM DJ. Quadriceps function, proprioceptive acuity and fuctional performance in healthy young, middle-aged and elderly subjects. Age Ageing 1998;27(1):55-62.

IBGE - Instituto Brasileiro de Geografia e Estatística. Síntese de Indicadores Sociais 2005. Disponível em:

www.ibge.gov.br/home/estatistica/populacao/condicaodevida/indicadoresmini $\underline{\text { mos/sinteseindicsociais2005/indic sociais2005.pdf }}$ Acessado 12 de dezembro de 2006. 
JUDGE JO, UNDERWOOD M, GENNOSA T. Exercise to improve gait velocity in older persons. Arch Phys Med Rehabil 1993;74:400- 406.

JUDGE JO, OUNPUU S, DAVIS RB. Effects of age on the biomechanics and physiology of gait. Clin Geriatr Med 1996:2(4): 659-678.

JUDGE JO, KENNY AM, KRAEMER WJ. Exercise in older adults. Conn Med 2003;67:461-464.

KERRIGAN DC, TODD MK, CROCE UD, LIPSITZ LA, COLLINS J. Biomechanical gait alterations independent of speed in the healthy elderly: evidence for specific limiting impairments. Arch Phys Med Rehabil 1998;79:317-322.

KREBS DE, SCARBOROUGH D M, MCGIBBONC. A. Functional vs. strength training in disabled elderly outpatients. Am J Phys Med Rehabil 2007;86:93103.

LAMOUREUX EL, SPARROW WA, MURPHY A, NEWTON RU. The relationship between lower body strength and obstructed gait in community dwelling older adults. J Am Geriatr Soc 2002;50(3): 468-473.

LANDERS KA, HUNTER GR, WETZSTEIN CJ, BAMMAN MM, WEINSIER $\mathrm{RL}$. The interrelationship among muscle mass, strength, and the ability to perform tasks of daily living in younger and older women. J Gerontol 2001;56A(10):B443-448.

LATHAM NK, BENNETT DA, STRETTON CM, ANDERSON CS. Systematic review of progressive resistance strength training in older adults. J Gerontol 2004;59A(1):48-61. 
LEE LW, ZAVAREI K, EVANS J, LALAS JJ, RILEY PO, KERRIGAN C. Reduced hip extension in the elderly: dynamic or postural? Arch Phys Med Rehabil 2005;86:1851-1854.

LEIPER CI, CRAIK RL. Relationships between physical activity and temporal-distance characteristics of walking in elderly women. Phys Ther 1991;71:791-803.

LIU MQ, ANDERSON FC, PANDY MG, DELP SL. Muscles that support the body also modulate forward progression during walking. J Biomech 2006;39:2623-2630.

LOPOPOLO RB, GRECO M SULLIVAN D, CRAIK RL, MANGIONE KK. Effect of therapeutic exercise on gait speed in community - dwelling elderly people: a meta- analysis. Phys Ther 2006;86(4):520-540.

MAKI BE, MCLROY WE. Control of rapid limb movements for balance recovery: age-related changes and implications for falls prevention. Age Ageing 2006;35-S2:ii12-18.

MARSICO V, MORETTI B, PATELLA V, SERIO S, SIMONE C. Analisi baropodometica del passo in soggetti sani anziani ed in pazienti gonadtrosici prima e dopo intervento di artroprotesi di ginocchio. G Ital Med Lav Erg 2002;24(1):72-83. Abstract.

McGIBBON CA. Toward a better understanding of gait changes with age and disablement: neuromuscular adaptation. Exerc Sport Sci Rev 2003;31(2):102-108.

MENZ HB, LORD SR, FITZPATRICK RC. Age - related differences in walking stability. Age Ageing 2003;32:137-142. 
MENZ HB, LORD SR, FITZPATRICK RC. A structural equation model relating impaired sensorimotor function, fear of falling and gait patterns in older people. Gait Posture 2007;25:243-249.

MUHLBERG W, SIEBER C. Sarcopenia and frailty in geriatric patient: implications for training and prevention. Z Gerontol Geriatr 2004;37 (1):2-8.

NEPTUNE RR, ZAJAC FE, KAUTZ S. A. Muscle force redistributes segmental power for body progression during walking. Gait Posture 2004;19:194-205.

OBERG T, KARSZNIA A, OBERG K. Basic gait parameters: reference data for normal subjects, 10-79 years of age. J Rehabil Res Dev 1993; 30(2):210223.

OSTIR GV, VOLPATO S, FRIED LP, CHAVES P, GURALNICK JM. Reliability and sensitivity to change assessed for a summary measure of lower body function results from the Women's health and aging study. J Clin Epidemiol 2002;55:916-921.

OSTROSKY KM, VANSWEARINGEN JM, BURDETT RG, GEE Z. A comparison of gait characteristics in young and old subjects. Phys Ther 1994;74:34-43.

OWINGS TM, GRABINER MD. Step width variability, but not step length variability or step time variability, discriminates gait of healthy young and older adults during treadmill locomotion. J Biomech 2003; 37:935-938.

OWINGS TM, GRABINER MD. Variability of step kinematics in young and older adults. Gait Posture 2004;20:26-29. 
PAPALÉO NETO M, CARVALHO FILHO ET. Geriatria: fundamentos, clínica e terapêutica, São Paulo: Atheneu, 2000.

PERRY, J. Análise de marcha: marcha normal. v.1. São Paulo: Manole, 2005.

PRINCE F, CORRIVEAU H, HÉBERT R, WINTER DA. Gait in the elderly. Gait Posture 1997;5:128-135.

PURSER JL, PIEPER CF, POOLE C, MOREY M. Trajectories of leg strength and gait speed among sedentary older adults: longitudinal pattern of dose response. J Gerontol2003;(58A),12:1125-1134.

REID S, SHAW A, HAUGH LD. Validity and reability of an internet based temporal gait assessment tool with healthy adults: a pilot study. Arch Phys Med Rehabil 2005;86:1014-1018.

REJESKI WJ, BRAWLEY LR. Med Sci Sports Exerc 2006;38:1,93-99.

RICHARD R, WEBER J, MEJJAD DP, DUJARDIN F, PASQUIS P, LE LOET X. Mesure des variables spatiotemporelles de la marche par le locometre de bessou en function de l'âge, de la taille et du sexe chez 79 sujets sains. Rev Rhum (ed. fr.) 1995;62(2),111-120.

RILEY PO, DELLACROCE U, KERRIGAN DC. Effect of age on lower extremity joint moment contributions to gait speed. Gait Posture 2001; 14:264-270.

RONSKY JL, NIGG BM, FISHER V. Correlation between physical activity and the gait characteristics and ankle joint flexibility of the elderly. Clin Biomech 1995;10:1,41-49. 
ROTH SM, IVEY FM, MARTEL GF, LEMMER JT, HURLBUT DE, SIEGEL $E L$, et al. Muscle size responses to strength training in young and older men and women. J Am Geriatr Soc 2001;49:1428-1433.

RUBINO FA. Gait disorders. Neurologist 2002;8:254-262.

SCARBOROUGH DM, KREBS DE, HARRIS BA. Quadriceps muscle strength and dynamic stability in elderly persons. Gait Posture 1999; 10:1020.

SEKIYA N, NAGASAKI H, ITO H, FURUNA T. Optimal walking in terms of variability in step length. J Orthop Sports Phys Ther 1997;26 (5):266-272.

SHKRURATOVA N, MORRIS ME, HUXHAM F. Effects of age on balance control during walking. Arch Phys Med Rehabil 2004;85(4): 582-588.

SILVESTRE JA, KALACHE A, RAMOS LR, VERAS RP. O envelhecimento populacional brasileiro e o setor saúde. Arq Geratr Gerontol 1996;(1):81-89.

SIPILA S, MULTANEM J, KALLINEN M, ERA P, SUOMINEN H. Effects of strength and endurance training on isometric muscle strength and walking speed in elderly women. Acta Physiol Scand 1996;156:457-464.

SIZÍNIO H, XAVIER R, PARDINI JAG, TARCICIO EPBF. Ortopedia e traumatologia - princípios e prática. $2^{2}$ ed., Porto Alegre: Artmed, 1998.

STEFFEN TM, HACKER TA, MOLLINGER L. Age and gender -related test performance in community - dwelling elderly people: six- minute walk test, berg balance scale, timed up \& go test and gait speeds. PhysTher 2002;82(2):128-137. 
STOLZE $H$, FRIEDRICH HJ, STEINAUER K, VIEREGGE P. Stride parameters in healthy young and old women - measurement variability on a simple walkway. Exp Aging Res 2000;26:159-168.

SULLIVAN SO, SCHMITZ TJ. Fisioterapia - Avaliação e tratamento. $2^{\text {a }}$ ed., São Paulo: Manole, 1993.

THOMPSON LV. Effects of age and training on skeletal muscle physiology and performance. Phys Ther 1994;74(1):71-81.

ZIJLSTRA W. Assessment of spatio-temporal parameters during unconstrained walking . Eur J Appl Physiol 2004;92:39-44. 


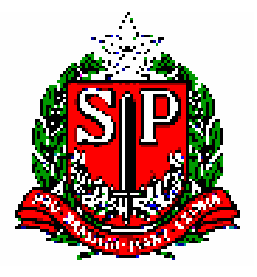

ANEXO I

\section{HOSPITAL DAS CLÍNICAS}

DA

FACULDADE DE MEDICINA DA UNIVERSIDADE DE SÃO PAULO

TERMO DE CONSENTIMENTO LIVRE E ESCLARECIDO

(Instruções para preenchimento no verso)

\section{I - DADOS DE IDENTIFICAÇÃO DO SUJEITO DA PESQUISA OU RESPONSÁVEL LEGAL}

1. NOME DO PACIENTE

DOCUMENTO DE IDENTIDADE № : SEXO : .M? F?

DATA NASCIMENTO: ........................

ENDEREÇO №

APTO:

BAIRRO:

CEP: TELEFONE:DDD

SEXO: M? F ?

2.RESPONSÁVEL LEGAL

NATUREZA (grau de parentesco, tutor, curador etc.)

DOCUMENTO DE IDENTIDADE : № APTO: ENDEREÇO: BAIRRO: CEP: ..CIDADE TELEFONE: DDD ... $\mathrm{N}$

\section{II - DADOS SOBRE A PESQUISA CIENTÍFICA}

\section{TÍTULO DO PROTOCOLO DE PESQUISA:}

Avaliação da marcha em pessoas idosas ativas e sedentárias. PESQUISADOR: Luciana Mastandrea CARGO/FUNÇÃO: fisioterapeuta INSCRIÇÃO CONSELHO REGIONAL №32316-F UNIDADE DO HCFMUSP: IOT

2. AVALIAÇÃO DO RISCO DA PESQUISA:

$\begin{array}{lll}\text { SEM RISCO } & ? & \text { RISCO MÍNIMO ? RISCO MÉdIO ? } \\ \text { RISCO BAIXO } & ? & \text { RISCO MAIOR ? }\end{array}$

3.DURAÇÃO DA PESQUISA : 24 meses

III - REGISTRO DAS EXPLICAÇÕES DO PESQUISADOR AO PACIENTE OU SEU REPRESENTANTE LEGAL SOBRE A PESQUISA, CONSIGNANDO:

Este estudo visa avaliar a maneira de você andar (sua marcha). Para a realização dos testes, será necessário que você venha com trajes confortáveis. Será realizada uma avaliação da maneira como você caminha e da velocidade de caminhar. Os resultados obtidos serão utilizados apenas com a finalidade de pesquisa, você não será identificado. Os testes não causarão desconforto ou prejuízo para você, não existe risco algum. A sua 
participação trará os benefícios de avaliar o ganho funcional e poder corrigir ou orientar o programa de treinamento realizado, pois o exame permite determinar as alterações funcionais da marcha. A qualquer momento você poderá desistir de participar desta pesquisa. Sua recusa não trará prejuízo ao tratamento atual, você não perderá o direito ou a qualidade no tratamento que esteja fazendo ou venha fazer no hospital. A sua participação não é obrigatória, porém será de muita importância para o desenvolvimento deste estudo. Se tiver de acordo com esses termos, por favor, assine abaixo.

IV - ESCLARECIMENTOS DADOS PELO PESQUISADOR SOBRE GARANTIAS DO SUJEITO DA PESQUISA

1. $\mathrm{O}$ (a) senhor(a) terá acesso, a qualquer tempo, às informações sobre procedimentos, riscos e benefícios relacionados à pesquisa, inclusive para dirimir eventuais dúvidas.

2. $\mathrm{O}$ (a) senhor (a) terá a liberdade de retirar seu consentimento a qualquer momento e de deixar de participar do estudo, sem que isto traga prejuízo à continuidade da assistência.

3. Garantiremos a salvaguarda da confidencialidade, sigilo e privacidade, mesmo no momento da publicação dos resultados.

4. $\mathrm{O}$ (a) senhor(a) terá disponibilidade de assistência no HCFMUSP, por eventuais danos à saúde, decorrentes da pesquisa.

5. $\mathrm{O}(\mathrm{a})$ senhor(a) não terá viabilidade de indenização por eventuais danos à saúde decorrentes da pesquisa.

6. $O$ (a) senhor(a) terá informação sobre qualquer nova descoberta relacionada a sua condição mesmo que possa afetar sua vontade de continuar participando da pesquisa.

\section{V- INFORMACÕES DE NOMES, ENDERECOS E TELEFONES DOS RESPONSÁVEIS PELO ACOMPANHAMENTO DA PESQUISA, PARA CONTATO EM CASO DE INTERCORRÊNCIAS CLÍNICAS E REAÇÕES ADVERSAS.}

Ft. Luciana Mastandrea (LEM) F: 3069-6041 / cel. 9107-0899

\section{Vl- OBSERVAÇÕES COMPLEMENTARES:}

\section{VII - CONSENTIMENTO PÓS-ESCLARECIDO}

Declaro que, após convenientemente esclarecido pelo pesquisador e ter entendido o que me foi explicado, consinto em participar do presente Protocolo de Pesquisa.

São Paulo, de de 
ANEXO II

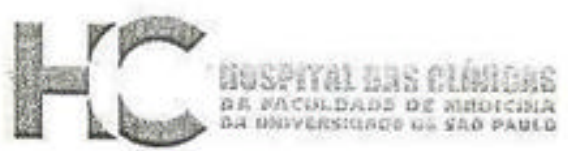

\section{APROVACÃO}

A Comissão de Ética para Análise de Projetos de Pesquisa - CAPPesq da Diretoria Clínica do Hospital das Clínicas e da Faculdade de Medicina da Universidade de São Paulo, em sessão de 24.11.05, APROVOU o Protocolo de Pesquisa $n^{\circ}$ 405/05, intitulado: "Avaliação da marcha e da aptidão física em pessoas idosas ativas e sedentárias" apresentado pelo Departamento de ORTOPEDIA E TRAUMATOLOGIA, inclusive o Termo de Consentimento Livre $e$ Esclarecido.

Cabe ao pesquisador elaborar e apresentar à CAPPesq, os relatórios parciais -e final sobre a pesquisa (Resolução do Conselho Nacional de Saúde $n^{\circ} 196$, de 10,10.1996, inciso IX. 2, letra "c")

Pesquisador(a) Responsável: Profa. Dra. Júlia Maria D'Andréa Greve Pesquisador (a) Executante: Sra. Luciana Mastandrea

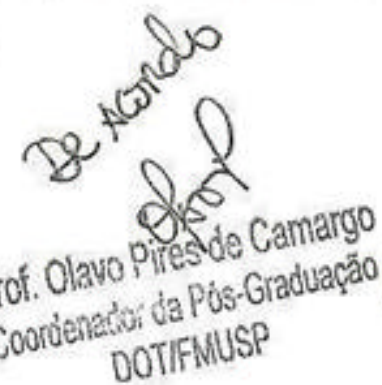

CAPPesq, 24 de Novembro de 2005.

$$
\text { lulde coxtlo }
$$

PROF. DR. EUCLIDES AYRES DE CASTILHO

Presidente da Comissão de Ética para Análise de Projetos de Pesquisa

Combssio de toka para Analise de Projetos de Pesquisa do HCFMUSP e da FMUSP Diretoria Cinica do Hospital das Cinicas da Faculdade de Medicina da Uatversidade de Sso Paulo

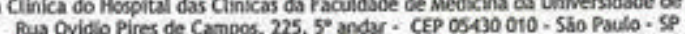

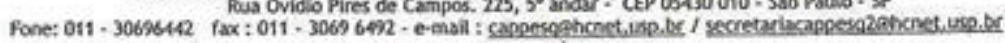


ANEXO III

\section{PROTOCOLO DE AVALIAÇÃO}

\section{IDENTIFICACÃO}

NOME:

DATA DE NASCIMENTO:

IDADE:

DATA DA AVALIAÇÃO: PROFISSÃO:

MEDICAMENTOS:

ATIVIDADE FÍSICA ATUAL:

( ) 1 x por semana ( ) $2 \times$ por semana ( ) 3x por semana ou mais

( ) sedentário

ATIVIDADE FÍSICA ANTERIOR:

( ) até 6 meses atrás ( ) até 1 ano atrás ( ) mais de 1 ano

\section{DADOS ANTROPOMÉTRICOS}

PESO:

ALTURA:

IMC:

\section{ANAMINESE}

QUEIXA (se houver):

ANTECEDENTES PESSOAIS E FAMILIARES:

LIMITAÇÃO NA VIDA DIÁRIA ( )Sim ( ) Não

PROBLEMAS VISUAIS ( )Sim ( ) Não

HIPOTENSÃO POSTURAL ( )Sim ( ) Não

ALTERAÇÕES DO SISTEMA VESTIBULAR ( ) Sim ( ) Não

FRATURAS DE MEMBROS INFERIORES CIRÚRGICAS PELO MENOS 3 ANOS ANTES DO ESTUDO ( ) Sim ( ) Não 
DOENÇAS QUE LIMITEM A PERMANÊNCIA EM POSIÇÃO ORTOSTÁTICA E A MARCHA ( )Sim ( ) Não QUEIXA DOLOROSA NOS PÉS IMPEDINDO LOCOMOÇÃO ( ) Sim ( ) Não

CAMINHA COM INDEPENDÊNCIA EM AMBIENTE EXTERNO POR PELO MENOS UM QUARTEIRÃO (100 m) E EM AMBIENTE INTERNO ( )Sim ( ) Não

\section{DOR}

( ) Sim ( ) Não LOCAL: 
APÊNDICE - Descrição das variáveis analisadas nos grupos Gerais (GER), Resistidos (RES) e Sedentários (SED).

\begin{tabular}{|c|c|c|c|c|c|c|c|c|c|}
\hline & \multirow[b]{2}{*}{$\mathbf{N}$} & \multirow[b]{2}{*}{ Média } & \multirow[b]{2}{*}{$\begin{array}{l}\text { Desvio } \\
\text { Padrão }\end{array}$} & \multirow[b]{2}{*}{$\begin{array}{l}\text { Erro } \\
\text { Padrão }\end{array}$} & \multicolumn{2}{|c|}{$\begin{array}{c}95 \% \text { Intervalo } \\
\text { Confiança por média }\end{array}$} & \multirow[b]{2}{*}{ Mínimo } & \multirow[b]{2}{*}{ Máximo } \\
\hline & & & & & & \begin{tabular}{l|} 
Lower \\
Bound
\end{tabular} & $\begin{array}{l}\text { Upper } \\
\text { Bound }\end{array}$ & & \\
\hline \multirow{4}{*}{ IDADE (anos) } & GER & 20 & 68,65 & 3,30 & 0,74 & 67,11 & 70,19 & 65,00 & 74,00 \\
\hline & RES & 20 & 69,05 & 2,91 & 0,65 & 67,69 & 70,41 & 65,00 & 74,00 \\
\hline & SED & 20 & 69,25 & 3,40 & 0,76 & 67,66 & 70,84 & 65,00 & 77,00 \\
\hline & Total & 60 & 68,98 & 3,16 & 0,41 & 68,17 & 69,80 & 65,00 & 77,00 \\
\hline \multirow{4}{*}{$\begin{array}{l}\text { TEMPO } \\
\text { PERCORRIDO } \\
4 \text { METROS (s) }\end{array}$} & GER & 20 & 3,24 & 0,22 & 0,05 & 3,13 & 3,34 & 2,85 & 3,68 \\
\hline & RES & 20 & 3,32 & 0,30 & 0,07 & 3,18 & 3,46 & 2,94 & 3,94 \\
\hline & SED & 20 & 3,87 & 0,40 & 0,09 & 3,69 & 4,06 & 3,10 & 4,55 \\
\hline & Total & 60 & 3,48 & 0,42 & 0,05 & 3,37 & 3,59 & 2,85 & 4,55 \\
\hline \multirow{4}{*}{$\begin{array}{l}\text { TEMPO } \\
\text { PERCORRIDO } \\
10 \text { METROS (s) }\end{array}$} & GER & 20 & 8,19 & 0,62 & 0,14 & 7,90 & 8,48 & 7,14 & 9,78 \\
\hline & RES & 20 & 8,01 & 0,65 & 0,15 & 7,71 & 8,31 & 6,69 & 9,15 \\
\hline & SED & 20 & 9,75 & 0,99 & 0,22 & 9,28 & 10,21 & 7,91 & 11,43 \\
\hline & Total & 60 & 8,65 & 1,09 & 0,14 & 8,37 & 8,93 & 6,69 & 11,43 \\
\hline \multirow{4}{*}{$\begin{array}{l}\text { TEMPO } \\
\text { PERCORRIDO } \\
10 \text { METROS } \\
\text { COM TINTA(s) }\end{array}$} & GER & 20 & 8,52 & 0,94 & 0,21 & 8,08 & 8,96 & 7,00 & 10,49 \\
\hline & RES & 20 & 8,30 & 0,77 & 0,17 & 7,94 & 8,67 & 6,99 & 9,99 \\
\hline & SED & 20 & 10,76 & 1,71 & 0,38 & 9,96 & 11,57 & 7,61 & 13,64 \\
\hline & Total & 60 & 9,20 & 1,64 & 0,21 & 8,77 & 9,62 & 6,99 & 13,64 \\
\hline \multirow{4}{*}{$\begin{array}{l}\text { VELOCIDADE } \\
4 \text { METROS }(\mathrm{m} / \mathrm{s})\end{array}$} & GER & 20 & 1,24 & 0,09 & 0,02 & 1,20 & 1,28 & 1,09 & 1,40 \\
\hline & RES & 20 & 1,21 & 0,10 & 0,02 & 1,16 & 1,26 & 1,01 & 1,36 \\
\hline & SED & 20 & 1,04 & 0,11 & 0,02 & 0,99 & 1,10 & 0,88 & 1,29 \\
\hline & Total & 60 & 1,17 & 0,13 & 0,02 & 1,13 & 1,20 & 0,88 & 1,40 \\
\hline \multirow{4}{*}{$\begin{array}{l}\text { VELOCIDADE } \\
10 \mathrm{METROS} \\
(\mathrm{m} / \mathrm{s})\end{array}$} & GER & 20 & 1,23 & 0,09 & 0,02 & 1,18 & 1,27 & 1,02 & 1,40 \\
\hline & RES & 20 & 1,26 & 0,11 & 0,02 & 1,21 & 1,31 & 1,09 & 1,49 \\
\hline & SED & 20 & 1,04 & 0,11 & 0,02 & 0,99 & 1,09 & 0,87 & 1,26 \\
\hline & Total & 60 & 1,17 & 0,14 & 0,02 & 1,14 & 1,21 & 0,87 & 1,49 \\
\hline
\end{tabular}




\begin{tabular}{|c|c|c|c|c|c|c|c|c|c|}
\hline \multirow{4}{*}{$\begin{array}{l}\text { VELOCIDADE } \\
10 \text { METROS } \\
\text { COM TINTA } \\
(\mathrm{m} / \mathrm{s})\end{array}$} & GER & 20 & 1,23 & 0,09 & 0,02 & 1,18 & 1,27 & 1,02 & 1,40 \\
\hline & RES & 20 & 1,26 & 0,11 & 0,02 & 1,21 & 1,31 & 1,09 & 1,49 \\
\hline & SED & 20 & 1,04 & 0,11 & 0,02 & 0,99 & 1,09 & 0,87 & 1,26 \\
\hline & Total & 60 & 1,17 & 0,14 & 0,02 & 1,14 & 1,21 & 0,87 & 1,49 \\
\hline \multirow{4}{*}{$\begin{array}{l}\text { COMPRIMENTO } \\
\text { SEIS PASSOS } \\
\text { (cm) }\end{array}$} & GER & 20 & 62,21 & 4,06 & 0,91 & 60,31 & 64,11 & 55,75 & 70,93 \\
\hline & \begin{tabular}{|l|} 
RES \\
\end{tabular} & 20 & 61,06 & 4,95 & 1,11 & 58,74 & 63,37 & 50,07 & 69,97 \\
\hline & SED & 20 & 55,04 & 7,07 & 1,58 & 51,73 & 58,35 & 43,75 & 68,37 \\
\hline & \begin{tabular}{|l|} 
Total \\
\end{tabular} & 60 & 59,43 & 6,27 & 0,81 & 57,81 & 61,06 & 43,75 & 70,93 \\
\hline \multirow{4}{*}{$\begin{array}{l}\text { COMPRIMENTO } \\
\text { SEIS } \\
\text { PASSADAS } \\
\text { (cm) }\end{array}$} & GER & 20 & 123,83 & 8,06 & 1,80 & 120,05 & 127,60 & 111,28 & 142,13 \\
\hline & \begin{tabular}{|l|} 
RES \\
\end{tabular} & 20 & 120,99 & 9,93 & 2,22 & 116,35 & 125,64 & 101,03 & 140,73 \\
\hline & SED & 20 & 110,96 & 14,17 & 3,17 & 104,33 & 117,59 & 86,83 & 137,72 \\
\hline & \begin{tabular}{|l|} 
Total \\
\end{tabular} & 60 & 118,59 & 12,18 & 1,57 & 115,45 & 121,74 & 86,83 & 142,13 \\
\hline \multirow{4}{*}{$\begin{array}{l}\text { LARGURA SEIS } \\
\text { PASSOS }(\mathrm{cm})\end{array}$} & GER & 20 & 6,93 & 1,79 & 0,40 & 6,09 & 7,77 & 2,98 & 9,80 \\
\hline & \begin{tabular}{|l} 
RES \\
\end{tabular} & 20 & 7,93 & 2,41 & 0,54 & 6,81 & 9,06 & 4,60 & 13,80 \\
\hline & \begin{tabular}{|l|} 
SED \\
\end{tabular} & 20 & 6,99 & 2,72 & 0,61 & 5,71 & 8,26 & 2,53 & 11,57 \\
\hline & \begin{tabular}{|l|} 
Total \\
\end{tabular} & 60 & 7,28 & 2,34 & 0,30 & 6,68 & 7,89 & 2,53 & 13,80 \\
\hline \multirow{4}{*}{$\begin{array}{l}\text { COMPRIMENTO } \\
\text { DEZ PASSOS } \\
\text { (cm) }\end{array}$} & GER & 20 & 62,14 & 4,21 & 0,94 & 60,17 & 64,11 & 55,70 & 71,27 \\
\hline & \begin{tabular}{|l|} 
RES \\
\end{tabular} & 20 & 60,77 & 4,82 & 1,08 & 58,51 & 63,03 & 50,71 & 70,21 \\
\hline & \begin{tabular}{|l|} 
SED \\
\end{tabular} & 20 & 55,68 & 7,08 & 1,58 & 52,36 & 58,99 & 43,93 & 69,64 \\
\hline & \begin{tabular}{|l|} 
Total \\
\end{tabular} & 60 & 59,53 & 6,10 & 0,79 & 57,95 & 61,10 & 43,93 & 71,27 \\
\hline \multirow{4}{*}{$\begin{array}{l}\text { COMPRIMENTO } \\
\text { DEZ PASSADAS } \\
\text { (cm) }\end{array}$} & GER & 20 & 124,39 & 8,40 & 1,88 & 120,46 & 128,32 & 111,28 & 142,42 \\
\hline & RES & 20 & 121,51 & 9,81 & 2,19 & 116,91 & 126,10 & 101,39 & 140,91 \\
\hline & \begin{tabular}{|l|} 
SED \\
\end{tabular} & 20 & 111,26 & 14,18 & 3,17 & 104,63 & 117,90 & 88,19 & 139,22 \\
\hline & \begin{tabular}{|l|} 
Total \\
\end{tabular} & 60 & 119,05 & 12,28 & 1,58 & 115,88 & 122,23 & 88,19 & 142,42 \\
\hline \multirow{4}{*}{$\begin{array}{l}\text { LARGURA DEZ } \\
\text { PASSOS (cm) }\end{array}$} & GER & 20 & 6,95 & 1,93 & 0,43 & 6,05 & 7,86 & 3,29 & 10,33 \\
\hline & \begin{tabular}{|l|} 
RES \\
\end{tabular} & 20 & 8,06 & 2,23 & 0,50 & 7,01 & 9,10 & 4,10 & 13,53 \\
\hline & \begin{tabular}{|l|} 
SED \\
\end{tabular} & 20 & 7,10 & 2,61 & 0,58 & 5,88 & 8,32 & 2,91 & 10,72 \\
\hline & \begin{tabular}{|l|} 
Total \\
\end{tabular} & 60 & 7,37 & 2,29 & 0,30 & 6,78 & 7,96 & 2,91 & 13,53 \\
\hline
\end{tabular}




\begin{tabular}{|l|l|r|r|r|r|r|r|r|r|}
\hline \multirow{4}{*}{$\begin{array}{l}\text { CADÊNCIA } \\
\text { (passos/min) }\end{array}$} & GER & 20 & 109,23 & 13,61 & 3,04 & 102,86 & 115,60 & 80,41 & 137,14 \\
\cline { 2 - 10 } & RES & 20 & 113,12 & 9,95 & 2,22 & 108,46 & 117,78 & 96,10 & 137,84 \\
\cline { 2 - 10 } & SED & 20 & 97,92 & 11,13 & 2,49 & 92,70 & 103,13 & 79,10 & 126,15 \\
\cline { 2 - 10 } & total & 60 & 106,76 & 13,18 & 1,70 & 103,35 & 110,16 & 79,10 & 137,84 \\
\hline \multirow{5}{*}{ ALTURA (cm) } & GER & 20 & 154,90 & 4,72 & 1,05 & 152,68 & 157,11 & 146,50 & 163,50 \\
\cline { 2 - 10 } & RES & 20 & 154,20 & 6,03 & 1,34 & 151,37 & 157,02 & 145,50 & 163,50 \\
\cline { 2 - 10 } & SED & 20 & 154,02 & 6,86 & 1,53 & 150,81 & 157,23 & 142,00 & 167,00 \\
\cline { 2 - 9 } & total & 60 & 154,37 & 5,85 & 0,75 & 152,86 & 155,88 & 142,00 & 169,00 \\
\hline \multirow{3}{*}{ IMC $\left(\mathrm{kg} / \mathrm{cm}^{2}\right)$} & GER & 20 & 27,57 & 4,22 & 0,94 & 25,59 & 29,54 & 18,15 & 33,08 \\
\cline { 2 - 9 } & RES & 20 & 26,39 & 3,19 & 0,71 & 24,89 & 27,88 & 20,89 & 33,61 \\
\cline { 2 - 9 } & SED & 20 & 26,49 & 4,05 & 0,90 & 24,59 & 28,39 & 19,86 & 32,82 \\
\cline { 2 - 9 } & total & 60 & 26,81 & 3,82 & 0,49 & 25,83 & 27,80 & 18,15 & 33,61 \\
\hline
\end{tabular}

